\title{
Preparation of Quinoline-2,4-dione Functionalized 1,2,3-Triazol-4-ylmethanols, 1,2,3-Triazole-4-carbaldehydes and 1,2,3-Triazole-4-carboxylic Acids
}

\author{
David Milićević, ${ }^{1}$ Roman Kimmel, ${ }^{1}$ Damijana Urankar, ${ }^{2}$ Andrej Pevec, ${ }^{2}$ \\ Janez Košmrlj ${ }^{2, *}$ and Stanislav Kafka ${ }^{1, *}$ \\ ${ }^{1}$ Faculty of Technology, Tomas Bata University in Zlin, Vavrečkova 275, 76001 Zlin, Czech Republic \\ ${ }^{2}$ Faculty of Chemistry and Chemical Technology, University of Ljubljana, Večna pot 113, SI-1000 Ljubljana, Slovenia \\ *Corresponding author: E-mail: kafka@utb.cz; Janez.Kosmrlj@fkkt.uni-lj.si \\ Tel: (+420)-576031115, (+386)-14798558
}

Received: 06-30-2019

Dedicated to Professor František Liška, University of Chemistry and Technology,

Prague and Charles University, on the occasion of his $80^{\text {th }}$ birthday.

\begin{abstract}
(1-(2,4-Dioxo-1,2,3,4-tetrahydroquinolin-3-yl)-1H-1,2,3-triazol-4-yl)methyl acetates substituted on nitrogen atom of quinolinedione moiety with propargyl group or (1-substituted $1 H-1,2,3$-triazol-4-yl)methyl group, which are available from the appropriate 3-(4-hydroxymethyl-1H-1,2,3-triazol-1-yl)quinoline-2,4 $(1 H, 3 H)$-diones unsubstituted on quinolone nitrogen atom by the previously described procedures, were deacetylated by acidic ethanolysis. Thus obtained primary alcohols, as well as those aforenamed unsubstituted on quinolone nitrogen atom, were oxidized to aldehydes on the one hand with pyridinium chlorochromate (PCC), on the other hand with manganese dioxide, and to carboxylic acids using Jones reagent in acetone. The structures of all prepared compounds were confirmed by ${ }^{1} \mathrm{H},{ }^{13} \mathrm{C}$ and ${ }^{15} \mathrm{~N}$ NMR spectroscopy. The corresponding resonances were assigned on the basis of the standard $1 \mathrm{D}$ and gradient selected $2 \mathrm{D}$ NMR experiments $\left({ }^{1} \mathrm{H}-{ }^{1} \mathrm{H}\right.$ gs-COSY, ${ }^{1} \mathrm{H}-{ }^{13} \mathrm{C}$ gs-HSQC, ${ }^{1} \mathrm{H}-{ }^{13} \mathrm{C}$ gs-HMBC) with ${ }^{1} \mathrm{H}-{ }^{15} \mathrm{~N}$ gs- $\mathrm{HMBC}$ as a practical tool to determine ${ }^{15} \mathrm{~N}$ NMR chemical shifts at the natural abundance level of ${ }^{15} \mathrm{~N}$ isotope.
\end{abstract}

Keywords: 1,2,3-triazole; quinoline-2,4-dione; hydroxymethylderivatives; aldehydes; carboxylic acids

\section{Introduction}

1,4-Disubstituted 1,2,3-triazole is considered to be a suitable structural part of compounds that could be of interest from the point of view of various research areas. Apart from many applicable properties including coordination ${ }^{1-3}$ and catalytic abilities, ${ }^{4}$ as well as photophysical and electrochemical characteristics, ${ }^{5-8}$ 1,2,3-triazoles further exhibit large variety of medical activities. ${ }^{9-14}$ Some of us have previously dealt with preparation of pyridine appended 1,2,3-triazoles and their synthetic utilization. ${ }^{15}$ 1,2,3-Triazolium salts prepared from them have shown an efficiency in palladium-catalyzed Suzuki-Miyaura coupling. ${ }^{15}$ From these salts, $\mathrm{Ru}$ (II) complexes were prepared, which have shown a catalytic activity in the oxidation of alcohols with tert-butyl hydroperoxide. ${ }^{16} \mathrm{Cp}^{*}-\mathrm{Ir}(\mathrm{III})$ complexes with additional chelating ligands containing 1,2,3-triazole ring are useful as catalysts for oxidation of cyclooctane to cyclooctanone. ${ }^{17} \mathrm{~A}$ bis(pyridyl-functionalized 1,2,3-triazol-5-ylidene)-palladium(II) complex $\left[\mathrm{Pd}(\mathrm{Py} \text {-tzNHC })_{2}\right]^{2+}$ was found to catalyze the copper-, amine-, phosphine-, and additive-free aerobic Sonogashira alkynylation of (hetero)aryl bromides in water as the only reaction solvent. ${ }^{18}$

Similarly, quinoline-2,4-dione based compounds were also recognized as distinctively attractive species, when taking into account their versatile beneficial purposefulness. ${ }^{19}$

The mentioned fact inspired us to synthesize never before described 1,2,3-triazole- and quinoline-2,4(1H,3H) dione-based bis-heterocycles. In 2011, we reported the synthesis of 3-alkyl/aryl-3-(1H-1,2,3-triazol-1-yl)quinoline-2,4(1H,3H)-diones by the click reaction of appropri- 
ate 3 -azidoquinolinediones with terminal alkynes. ${ }^{20}$ In the same year, the patent application ${ }^{21}$ was administered, which comprised preparation of tautomeric 4-hydroxyquinolin-2-ones with 1,2,3-triazol-1-yl group in position 3 of quinolinone scaffold that are effective as adenosine monophosphate-activated protein kinase (AMPK) activators. Since then still more reports on the 4-hydroxy-quinolin-2-one derivatives, in which hydrogen atom of hydroxyl group was replaced with various substituents comprising 1,2,3-triazole pattern, have been published. ${ }^{22-29}$ Some of these substances have been found to show some acetylcholine receptors binding affinity. ${ }^{22}$ Recently we have reported an utilization of the above mentioned 3-(1H-1,2,3-triazol1-yl)quinoline-2,4(1H,3H)-diones unsubstituted on the nitrogen atom of the quinolone moiety for the synthesis of bis(1,2,3-triazole) functionalized quinoline-2,4-diones.30 In frame of that study, ${ }^{30}$ in place of starting materials were used, among others, derivatives of ( $1 H$-1,2,3-triazol-4-yl) methanol, in which hydroxyl group was protected by acetylation and their structure was subsequently modified. There was offered the idea of the removal of protecting acetyl group and the oxidative conversion of thus obtained primary alcohols as well as starting triazolylmethanols to the corresponding 1,2,3-triazole-4-carbaldehydes and 1,2,3-triazole-4-carboxylic acids.

In terms of biological effects, 1,2,3-triazole-4-carbaldehydes are particularly interesting. For example, a series of them has been found to prove tuberculostatic effect. ${ }^{31}$ Some (1H-1,2,3-triazol-4-yl)methanols exhibit cytotoxic activity. ${ }^{32}$ Some known 1-substituted 1,2,3-triazol-4-carboxylic acids have antibacterial effect against Staphylococcus aureus. ${ }^{33}$

\section{Results and Discussion}

Compounds 1, 2 and 3 (Figure 1) were obtained through the multistep synthetic pathway, which we have developed recently, ${ }^{30}$ and were utilized as starting compounds in this study.

Although acetates 1a,b were prepared by acetylation of the corresponding primary alcohols $\mathbf{4 a}, \mathbf{b},{ }^{30}$ we exploited them as model compounds and dealt with finding a suitable procedure for their deacetylation back to the mentioned alcohols so that we can apply it to prepare alcohols $\mathbf{5 a}, \mathbf{b}$ and $\mathbf{6 a}-\mathbf{f}$ from the more laboriously obtainable corresponding acetates $\mathbf{2} \mathbf{a}, \mathbf{b}$ and $\mathbf{3 a} \mathbf{a}-\mathbf{f}$. At first, we tried processing with a methanolic solution of sodium methoxide, however, in parallel with ester methanolysis, undesirable nucleophilic quinoline-2,4-dione ring opening and successive reactions took place resulting in mixtures, from which only corresponding $N$-substituted anthranilic acids and eventually their methyl esters were isolated after neutralization with diluted hydrochloric acid. Also alkaline hydrolysis of ester group is accompanied with above mentioned ring opening; the treatment of $\mathbf{1 b}$ with a solution of potassium hydroxide in aqueous ethanol afforded corresponding anthranilic acid as main product.

Finally, acidic alcoholysis $(37 \% \mathrm{HCl}: \mathrm{EtOH}$ 1:100 $\mathrm{v} / \mathrm{v}$ ) has proved to be suitable. After the successful deacetyl-

Table 1. Acidic alcoholysis of acetates $\mathbf{1 a}, \mathbf{b}, \mathbf{2 a}, \mathbf{b}$, and $\mathbf{3 a} \mathbf{a}-\mathbf{f}$.

\begin{tabular}{ccccccc}
\hline Entry & Acetate & $\mathbf{R}^{\mathbf{1}}$ & $\mathbf{R}^{\mathbf{2}}$ & Time (h) & Alcohol & Yield (\%) \\
\hline 1 & $\mathbf{1 a}$ & $\mathrm{Me}$ & - & 3 & $\mathbf{4 a}$ & $92^{\mathrm{a}}$ \\
2 & $\mathbf{1 b}$ & $\mathrm{Ph}$ & - & 3 & $\mathbf{4 b}$ & $93^{\mathrm{a}}$ \\
3 & $\mathbf{2 a}$ & $\mathrm{Me}$ & - & 3 & $\mathbf{5 a}$ & $83^{\mathrm{b}}$ \\
4 & $\mathbf{2 b}$ & $\mathrm{Ph}$ & - & 3 & $\mathbf{5 b}$ & $87^{\mathrm{b}}$ \\
5 & $\mathbf{3 a}$ & $\mathrm{Me}$ & $\mathrm{Bn}$ & 3.5 & $\mathbf{6 a}$ & $86^{\mathrm{a}}$ \\
6 & $\mathbf{3 b}$ & $\mathrm{Me}$ & $\mathrm{Ph}$ & 3.5 & $\mathbf{6 b}$ & $98^{\mathrm{a}}$ \\
7 & $\mathbf{3 c}$ & $\mathrm{Me}$ & $2-\mathrm{Py}$ & 2.5 & $\mathbf{6 c}$ & $80^{\mathrm{a}}$ \\
8 & $\mathbf{3 d}$ & $\mathrm{Ph}$ & $\mathrm{Bn}$ & 4 & $\mathbf{6 d}$ & $89^{\mathrm{a}}$ \\
9 & $\mathbf{3 e}$ & $\mathrm{Ph}$ & $\mathrm{Ph}$ & 3 & $\mathbf{6 e}$ & $97^{\mathrm{a}}$ \\
10 & $\mathbf{3 f}$ & $\mathrm{Ph}$ & $2-\mathrm{Py}$ & 3 & $\mathbf{6 f}$ & $87^{\mathrm{a}}$ \\
\hline
\end{tabular}

${ }^{a}$ Refers to pure (by TLC and IR) isolated product. ${ }^{\mathrm{b}}$ Refers to percent yield of crystallized product.<smiles>[R]C1(n2cc(Cl)nn2)C(=O)Nc2ccccc2C1=O</smiles>

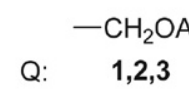<smiles>[R16]C1(n2cc(O)nn2)C(=O)c2ccccc2N(CC#C)C1=O</smiles>

$2,5,8,11$<smiles>CC[AsH3+]</smiles>

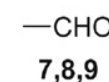<smiles>[R]n1cc(CN2C(=O)C([R])(n3cc(O)nn3)C(=O)c3ccccc32)nn1</smiles>

$-\mathrm{COOH}$

$10,11,12$

$$
\begin{array}{r|ll}
\mathbf{1 , 2 , 4 , 5}, & & \\
7,8,10,11 & \text { a } & \text { b } \\
\hline \mathrm{R}^{1} & \mathrm{Me} & \mathrm{Ph}
\end{array}
$$

\begin{tabular}{r|llllll}
$\mathbf{3}, \mathbf{6}$, & & & & & & \\
$\mathbf{9 , 1 2}$ & a & b & c & d & e & f \\
\hline $\mathrm{R}^{1}$ & $\mathrm{Me}$ & $\mathrm{Me}$ & $\mathrm{Me}$ & $\mathrm{Ph}$ & $\mathrm{Ph}$ & $\mathrm{Ph}$ \\
\hline $\mathrm{R}^{2}$ & $\mathrm{Bn}$ & $\mathrm{Ph}$ & $2-\mathrm{Py}$ & $\mathrm{Bn}$ & $\mathrm{Ph}$ & $2-\mathrm{Py}$
\end{tabular}

Figure 1. Subject compounds 
ation of compounds $\mathbf{1 a}, \mathbf{b}$, this method was applied also to deacetylation the acetates $\mathbf{2 a}, \mathbf{b}$ and $\mathbf{3 a} \mathbf{a}-\mathbf{f}$. This reaction was carried out by boiling the reaction mixtures and was finished in 2.5-4 hours. The appropriate primary alcohols were obtained with yields $80-90 \%$ (see Table 1).

The reaction conditions of the conversion of prepared alcohols to the corresponding aldehydes were optimized for the oxidation of alcohols $\mathbf{4 a}, \mathbf{b}$ to aldehydes $\mathbf{7 a}, \mathbf{b}$. The results are summarized in the Table 2. From the range of usual reagents used for these transformations, we first chose pyridinium chlorochromate (PCC). As can be found in literature, 34 oxidations of primary alcohol to aldehydes can proceed smoothly with good yields using $1.2 \mathrm{mmol}$ PCC per $1 \mathrm{mmol}$ substrate in dichloromethane (DCM) at room temperature. However, the conversion of $\mathbf{4 a}$ under these conditions is very slow, because of its low solubility in DCM. Boiling the reaction mixture, and particularly by performing the reaction in a microwave reactor in a closed vial at $40^{\circ} \mathrm{C}$, the time required to react the substrate is significantly reduced, but at the same time decreases the yield of 7a. Higher yields of 7a were achieved when DCM was replaced with acetone, in which $\mathbf{4 a}$ is more soluble; we have achieved the best yield (36\%) of $7 \mathbf{a}$ by increasing the excess of PCC and allowing the reaction to proceed for 22 hours at room temperature.

Oxidation of $\mathbf{4 b}$, which is more soluble in DCM than its methyl analogue $\mathbf{4 a}$, was performed in this solvent with the best yield (44\%) of $\mathbf{7 b}$ using $1.2 \mathrm{mmol}$ PCC per $1 \mathrm{mmol}$ $\mathbf{4 b}$ and boiling of the reaction mixture, whereas the reaction was finished within 1.5 hour. When the mixture of the same initial composition was heated in a microwave reactor in a closed vial to $40{ }^{\circ} \mathrm{C}$ for 10 minutes, the yield of $\mathbf{7 b}$ was only slightly lower than the former. The same applies to carrying out the reaction in DCM with $1.5 \mathrm{mmol}$ PCC per $1 \mathrm{mmol} \mathbf{4 b}$ at room temperature. Further increasing of the amount of PCC results in a shorter reaction time together with a reduction of yield of $7 \mathbf{b}$. In contrast to oxidation of $4 \mathbf{a}$ to $7 \mathbf{a}$, the oxidation of $4 \mathbf{b}$ with PCC in acetone under the same conditions furnished the aldehyde $\mathbf{7 b}$ with significantly lower yield.

Apart from oxidation with PCC, Swern reaction, i.e. oxidation with dimethylsulfoxide (DMSO) in the presence of oxalyl chloride and $\mathrm{N}, \mathrm{N}$-diisopropylethylamine (DIPEA), was also briefly examined using slightly modified synthetic procedure from the literature, ${ }^{35}$ however obtained yields were unsatisfactory for both, phenyl and methyl mono-triazole derivatives $7 \mathbf{a}$ and $\mathbf{7 b}$, respectively. While the former resulted in 33\% yield of isolated product, no product was isolated in case of the latter. The main drawback of this approach is presence of hardly removable dimethyl sulfoxide that remained in our products despite the fact that they were several times washed with ice-cold water. Apparently, utilization of relatively large quantities of water also caused significant loses of target compounds that were much more obvious in the case of methyl derivative 7a. Moreover, we have experience that our 1,2,3-triazole- and quinoline-2,4-dione-based bis-heterocycles are more or less unstable in DMSO and therefore, the use of this solvent in their preparation is not always appropriate.

As the third option, oxidation of primary alcohols 4a,b with $\mathrm{MnO}_{2}$ was further studied. Comparing the reaction parameters such as reaction times and quantities of reagents, acetone was recognized superior in comparison

Table 2. Oxidation of primary alcohols $\mathbf{4 a}, \mathbf{b}$ to aldehydes $7 \mathbf{a}, \mathbf{b} .^{\text {a }}$

\begin{tabular}{|c|c|c|c|c|c|c|c|c|}
\hline Entry & Alcohol & $\mathbf{R}^{1}$ & Reagent & $n$ (mmol) $^{\mathbf{b}}$ & Time (h) & Solvent & Aldehyde & Yield (\%) \\
\hline 1 & $4 a$ & $\mathrm{Me}$ & PCC & 1.7 & $22^{c}$ & $\mathrm{Me}_{2} \mathrm{CO}$ & $7 \mathbf{a}$ & $36^{\mathrm{d}}$ \\
\hline 2 & $4 a$ & $\mathrm{Me}$ & PCC & 1.2 & $22^{c}$ & $\mathrm{CH}_{2} \mathrm{Cl}_{2}$ & $7 \mathbf{a}$ & $31^{d}$ \\
\hline 3 & $4 a$ & $\mathrm{Me}$ & PCC & 1.2 & $0.17^{\mathrm{e}}$ & $\mathrm{CH}_{2} \mathrm{Cl}_{2}$ & $7 \mathbf{a}$ & 16 \\
\hline 4 & $4 a$ & $\mathrm{Me}$ & PCC & 1.2 & 1.5 & $\mathrm{CH}_{2} \mathrm{Cl}_{2}$ & $7 \mathbf{a}$ & 15 \\
\hline 5 & $4 a$ & $\mathrm{Me}$ & PCC & 1.2 & 5 & $\mathrm{Me}_{2} \mathrm{CO}$ & $7 \mathbf{a}$ & $23^{d}$ \\
\hline 6 & $4 b$ & $\mathrm{Ph}$ & PCC & 1.7 & $1.5^{\mathrm{c}}$ & $\mathrm{CH}_{2} \mathrm{Cl}_{2}$ & $7 \mathbf{b}$ & 35 \\
\hline 7 & $4 b$ & $\mathrm{Ph}$ & PCC & 1.7 & $22^{c}$ & $\mathrm{Me}_{2} \mathrm{CO}$ & $7 \mathbf{b}$ & $26^{\mathrm{d}}$ \\
\hline 8 & $4 b$ & $\mathrm{Ph}$ & PCC & 1.7 & 0.5 & $\mathrm{CH}_{2} \mathrm{Cl}_{2}$ & $7 \mathbf{b}$ & 34 \\
\hline 9 & $4 b$ & $\mathrm{Ph}$ & PCC & 2.0 & $1^{\mathrm{c}}$ & $\mathrm{CH}_{2} \mathrm{Cl}_{2}$ & $7 \mathbf{b}$ & 31 \\
\hline 10 & $4 b$ & $\mathrm{Ph}$ & PCC & 1.5 & $4^{c}$ & $\mathrm{CH}_{2} \mathrm{Cl}_{2}$ & $7 \mathbf{b}$ & 41 \\
\hline 11 & $4 b$ & $\mathrm{Ph}$ & PCC & 1.2 & 1.5 & $\mathrm{CH}_{2} \mathrm{Cl}_{2}$ & $7 \mathbf{b}$ & 44 \\
\hline 12 & $4 b$ & $\mathrm{Ph}$ & PCC & 1.2 & $0.17^{\mathrm{e}}$ & $\mathrm{CH}_{2} \mathrm{Cl}_{2}$ & $7 \mathbf{b}$ & 42 \\
\hline 13 & $4 a$ & $\mathrm{Me}$ & DMSO & 2.6 & $3.5^{\mathrm{f}}$ & $\mathrm{Me}_{2} \mathrm{CO}$ & $7 \mathbf{a}$ & 0 \\
\hline 14 & $4 b$ & $\mathrm{Ph}$ & DMSO & 2.6 & $3.5^{\mathrm{f}}$ & $\mathrm{CH}_{2} \mathrm{Cl}_{2}$ & $7 \mathbf{b}$ & 33 \\
\hline 15 & $4 a$ & $\mathrm{Me}$ & $\mathrm{MnO}_{2}$ & 10 & 1.25 & $\mathrm{Me}_{2} \mathrm{CO}$ & $7 \mathbf{a}$ & 60 \\
\hline 16 & $4 b$ & $\mathrm{Ph}$ & $\mathrm{MnO}_{2}$ & 10 & 1.5 & $\mathrm{Me}_{2} \mathrm{CO}$ & $7 \mathbf{b}$ & 58 \\
\hline 17 & $4 b$ & $\mathrm{Ph}$ & $\mathrm{MnO}_{2}$ & $15^{\mathrm{g}}$ & 3 & $\mathrm{CH}_{2} \mathrm{Cl}_{2}$ & $7 \mathbf{b}$ & 62 \\
\hline 18 & $4 b$ & $\mathrm{Ph}$ & $\mathrm{MnO}_{2}$ & 10 & $96^{c}$ & $\mathrm{CH}_{2} \mathrm{Cl}_{2}$ & $7 \mathbf{b}$ & 44 \\
\hline
\end{tabular}

${ }^{a}$ Reactions were carried out in boiling reaction mixtures unless indicated otherwise. ${ }^{\mathrm{b}}$ Amount of reagent per $1 \mathrm{mmol}$ of alcohol. ${ }^{\mathrm{c}} \mathrm{Carried}$ out at room temperature. ${ }^{\mathrm{d}}$ Complete consumption of $\mathbf{4}$ was not reached. ${ }^{\mathrm{e}}$ Carried out in the microwave reactor at $40{ }^{\circ} \mathrm{C} .{ }^{\mathrm{f}}$ For the reaction conditions see Experimental. ${ }^{\mathrm{g}}$ Reaction was started with $10 \mathrm{mmol}$ of $\mathrm{MnO}_{2}$, additional $5 \mathrm{mmol}$ of $\mathrm{MnO}_{2}$ were added after 2 hours. 
Table 3. Oxidation of primary alcohols $\mathbf{5 a}, \mathbf{b}$ and $\mathbf{6 a}-\mathbf{f}$ to aldehydes $\mathbf{8 a}, \mathbf{b}$ and $\mathbf{9 a}-\mathbf{f}$, respectively.

\begin{tabular}{|c|c|c|c|c|c|c|c|c|}
\hline Entry & Alcohol & $\mathbf{R}^{1}$ & $\mathbf{R}^{2}$ & Reagent & Time (h) & Solvent & Aldehyde & Yield (\%) \\
\hline 1 & $5 a$ & $\mathrm{Me}$ & - & PCC & 1 & $\mathrm{CH}_{2} \mathrm{Cl}_{2}$ & $8 a$ & 41 \\
\hline 2 & $5 a$ & $\mathrm{Me}$ & - & $\mathrm{MnO}_{2}$ & 1.25 & $\mathrm{Me}_{2} \mathrm{CO}$ & $8 a$ & 40 \\
\hline 3 & $5 b$ & $\mathrm{Ph}$ & - & PCC & 0.75 & $\mathrm{CH}_{2} \mathrm{Cl}_{2}$ & $8 b$ & 38 \\
\hline 4 & $5 \mathbf{b}$ & $\mathrm{Ph}$ & - & $\mathrm{MnO}_{2}$ & 2 & $\mathrm{Me}_{2} \mathrm{CO}$ & $8 b$ & 38 \\
\hline 5 & $6 a$ & $\mathrm{Me}$ & $\mathrm{Bn}$ & PCC & 0.5 & $\mathrm{CH}_{2} \mathrm{Cl}_{2}$ & $9 a$ & 41 \\
\hline 6 & $6 b$ & $\mathrm{Me}$ & $\mathrm{Ph}$ & PCC & 0.5 & $\mathrm{CH}_{2} \mathrm{Cl}_{2}$ & $9 b$ & 40 \\
\hline 7 & 6b & $\mathrm{Me}$ & $\mathrm{Ph}$ & $\mathrm{MnO}_{2}$ & 0.75 & $\mathrm{Me}_{2} \mathrm{CO}$ & $9 b$ & 51 \\
\hline 8 & $6 c$ & $\mathrm{Me}$ & 2-Py & PCC & 0.75 & $\mathrm{CH}_{2} \mathrm{Cl}_{2}$ & $9 c$ & 48 \\
\hline 9 & $6 d$ & $\mathrm{Ph}$ & $\mathrm{Bn}$ & PCC & 0.5 & $\mathrm{CH}_{2} \mathrm{Cl}_{2}$ & 9d & 41 \\
\hline 10 & $6 e$ & $\mathrm{Ph}$ & $\mathrm{Ph}$ & PCC & 0.5 & $\mathrm{CH}_{2} \mathrm{Cl}_{2}$ & $9 e$ & 45 \\
\hline 11 & $6 f$ & $\mathrm{Ph}$ & 2-Py & PCC & 0.5 & $\mathrm{CH}_{2} \mathrm{Cl}_{2}$ & 9f & 41 \\
\hline
\end{tabular}

with dichloromethane, while transformation yields were practically the same (approx. 60\%) in both cases.

The findings from the above experiments were used in the oxidation of primary alcohols $\mathbf{5 a}, \mathbf{b}$ and $\mathbf{6 a}-\mathbf{f}$ to aldehydes $\mathbf{8 a}, \mathbf{b}$ and $\mathbf{9 a - f}$, respectively. In all cases, oxidation was carried out using PCC under optimum conditions for the conversion of alcohol $\mathbf{4 b}$ to aldehyde $\mathbf{7 b}$, i.e. using 1.2 mmol PCC per $1 \mathrm{mmol}$ alcohol in dichloromethane at the reflux temperature. Furthermore, the aldehydes $\mathbf{8 a}, \mathbf{b}$ and $\mathbf{9 b}$ were prepared from the corresponding alcohols by oxidation with $\mathrm{MnO}_{2}$ in acetone. Due to the very similar yields of aldehydes achieved with the use of one or the other reagent and the toxicity of $\mathrm{Cr}^{\mathrm{VI}}$-containing reagents, it can be stated that $\mathrm{MnO}_{2}$ is a more advantageous agent than PCC.

So far described oxidations of triazolyl-4-methanols to the corresponding carboxylic acids were carried out mostly with permanganate in basic medium. ${ }^{36-38}$ In one case, the oxidation with a mixture of sodium chlorite and sodium hypochlorite with an addition of 2,2,6,6-tetramethylpiperidine $\mathrm{N}$-oxide (TEMPO) in phosphate buffer was patented. ${ }^{39}$ Since basic media causes destruction of quinolinedione scaffold, the choice of reagents for the oxidation of alcohols $\mathbf{4 a}, \mathbf{b}, \mathbf{5 a}, \mathbf{b}$, and $\mathbf{6 a}-\mathbf{f}$ is limited to those, for which the presence of no base is needed. For the transformation of these alcohols to carboxylic acids 10a,b, $11 \mathbf{a}, \mathbf{b}$, and 12a-f, we decided to try out Jones reagent (solution of $\mathrm{CrO}_{3}$ in diluted sulfuric acid) in acetone. While this method has long been known and its use for the preparation of carboxylic acids has been described in many cases, we have found in the literature only one report ${ }^{40}$ on its use for the preparation of triazole-4-carboxylic acids, which were intermediates in a multistep synthesis, without giving their yields and experimental details. Although at most $9 \mathrm{~mol}$ of $\mathrm{CrO}_{3}$ per one mol of primary alcohol is usually used, ${ }^{41-43}$ in the cases provided herein, it has been shown that the most suitable ratio is $24 \mathrm{~mol} \mathrm{CrO}_{3}$ per $1 \mathrm{~mol}$ of primary alcohol (Table 4). The acid with methyl group in position 3 of quinolone scaffold 10a was isolated in a considerably lower yield than its phenyl ana-
Table 4. Oxidation of primary alcohols $4 \mathbf{a}, \mathbf{b}, \mathbf{5 a}, \mathbf{b}$, and $\mathbf{6 a}-\mathbf{f}$ to carboxylic acids 10a,b, 11a,b, and 12a-f, respectively ${ }^{\mathrm{a}}$.

\begin{tabular}{ccccccc}
\hline Entry & Alcohol & $\mathbf{R}^{\mathbf{1}}$ & $\mathbf{R}^{\mathbf{2}}$ & Time (h) & $\begin{array}{c}\text { Carboxylic } \\
\text { acid }\end{array}$ & Yield (\%) \\
\hline 1 & & & & & $\mathbf{1 0 a}$ & 33 \\
2 & $\mathbf{4 b}$ & $\mathrm{Me}$ & - & 2.75 & $\mathbf{1 0 a}$ & $40^{\mathrm{b}}$ \\
3 & $\mathbf{4 b}$ & $\mathrm{Ph}$ & - & 3 & $\mathbf{1 0 b}$ & 71 \\
4 & $\mathbf{5 a}$ & $\mathrm{Me}$ & - & 2.5 & $\mathbf{1 1 a}$ & 55 \\
5 & $\mathbf{5 b}$ & $\mathrm{Ph}$ & - & 3 & $\mathbf{1 1 b}$ & 68 \\
6 & $\mathbf{6 a}$ & $\mathrm{Me}$ & $\mathrm{Bn}$ & 3 & $\mathbf{1 2 a}$ & 88 \\
7 & $\mathbf{6 b}$ & $\mathrm{Me}$ & $\mathrm{Ph}$ & 2.5 & $\mathbf{1 2 b}$ & 92 \\
8 & $\mathbf{6 c}$ & $\mathrm{Me}$ & $2-\mathrm{Py}$ & 2.5 & $\mathbf{1 2 c}$ & 84 \\
9 & $\mathbf{6 d}$ & $\mathrm{Ph}$ & $\mathrm{Bn}$ & 2 & $\mathbf{1 2 d}$ & 75 \\
10 & $\mathbf{6 e}$ & $\mathrm{Ph}$ & $\mathrm{Ph}$ & 2.25 & $\mathbf{1 2 e}$ & 77 \\
11 & $\mathbf{6 f}$ & $\mathrm{Ph}$ & $2-\mathrm{Py}$ & 2.5 & $\mathbf{1 2 f}$ & 69 \\
\hline
\end{tabular}

a $\mathrm{CrO}_{3}(2.4 \mathrm{~g}, 24 \mathrm{mmol})$ in $2 \mathrm{M} \mathrm{H}_{2} \mathrm{SO}_{4}(24 \mathrm{~mL})$ per $1 \mathrm{mmol}$ of alcohol was used, unless otherwise stated. ${ }^{\mathrm{b}} \mathrm{CrO}_{3}(600 \mathrm{mg}, 6.0$ mmol) in $2 \mathrm{M} \mathrm{H}_{2} \mathrm{SO}_{4}(6 \mathrm{~mL})$ per $1 \mathrm{mmol}$ of alcohol $4 \mathbf{b}$ was used, complete consumption of an intermediate (apparently aldehyde 7b) was not reached according to TLC.

logue $\mathbf{1 0 b}$ probably due to its significantly higher solubility in water.

All compounds were characterized by ${ }^{1} \mathrm{H}$ and ${ }^{13} \mathrm{C}$ and, in cases of $\mathbf{6 a}-\mathbf{e}, 7 \mathbf{a}, \mathbf{b}, 9 \mathbf{a}-\mathbf{f}, 10 \mathbf{a}$, and $12 \mathbf{a}-\mathbf{f}$, also by ${ }^{15} \mathrm{~N}$ NMR spectroscopy. The corresponding resonances
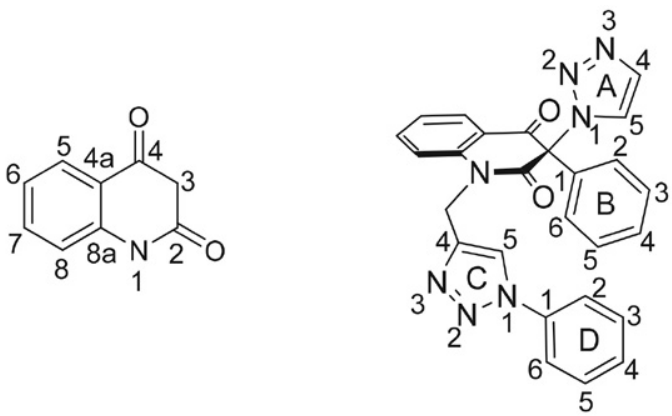

Figure 2. Designation of positions in the structure of prepared compounds. 
were assigned on the basis of gradient-selected 2D NMR experiments including ${ }^{1} \mathrm{H}-{ }^{1} \mathrm{H}$ gs-COSY, ${ }^{1} \mathrm{H}-{ }^{13} \mathrm{C}$ gsHSQC, ${ }^{1} \mathrm{H}-{ }^{13} \mathrm{C}$ gs-HMBC and ${ }^{1} \mathrm{H}-{ }^{15} \mathrm{~N}$ gs-HMBC. Atoms and rings labeling scheme, which was extensively applied in the »Experimental « section is presented in Figure 2.

From the solution of $\mathbf{1 2 d}$ in deuteriochloroform originally designed to measure NMR spectra, the crystal has grown, which we have used to corroborate the structure of this compound (Figure 3) by the single crystal $\mathrm{X}$-ray structure determination. It has been found that the crystal is a solvate $\mathbf{1 2 d} \cdot 2 \mathrm{CDCl}_{3}$. Selected bond lengths and angles are displayed in Table 5. The X-ray diffraction study has shown that the solvate $\mathbf{1 2 d} \cdot 2 \mathrm{CDCl}_{3}$ crystallizes

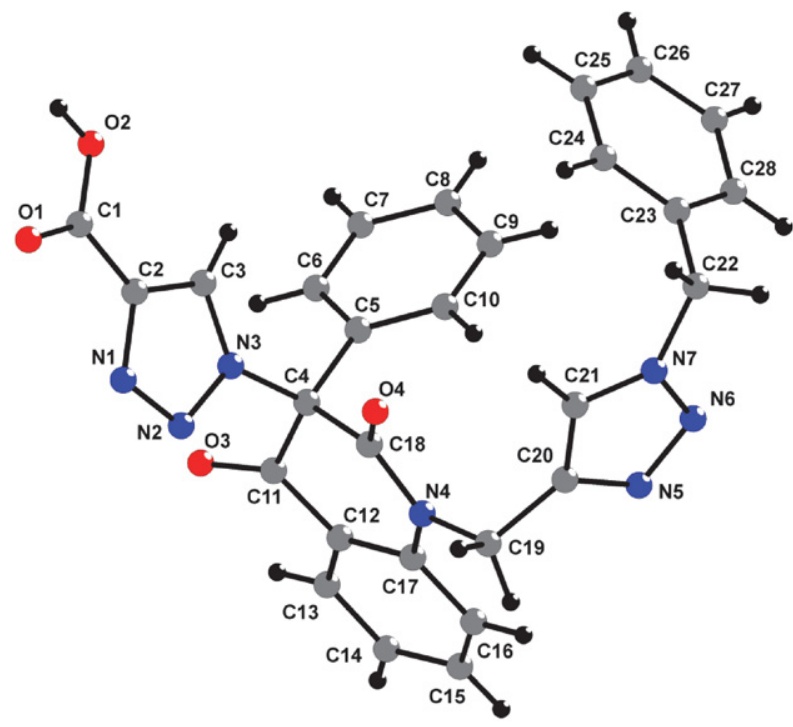

Figure 3. Crystallographic view and numbering scheme of the molecule $12 \mathrm{~d} \cdot 2 \mathrm{CDCl}_{3}$. $\mathrm{CDCl}_{3}$ molecules are omitted for clarity. in monoclinic $P 2_{1} / n$ space group. Intermolecular hydrogen bonds of the type $\mathrm{O}-\mathrm{H}$... N are found in the crystal structure of compound $\mathbf{1 2 d} \cdot 2 \mathrm{CDCl}_{3}$. Atom $\mathrm{O} 2$ acts as hydrogen bond donor and $\mathrm{N} 5$ of symmetry related molecule as acceptor and thus forming two dimensional chain extending along the $b$-axis (Figure 4 , Table 6).

Table 5. Selected bond lengths $(\AA)$ and angles $\left(^{\circ}\right)$ for compound $12 \mathrm{~d} \cdot 2 \mathrm{CDCl}_{3}$

\begin{tabular}{llll}
\hline N1-N2 & $1.298(5)$ & N1-N2-N3 & $106.8(3)$ \\
N2-N3 & $1.359(5)$ & N5-N6-N7 & $106.8(4)$ \\
N5-N6 & $1.310(5)$ & N2-N3-C3 & $110.7(3)$ \\
N6-N7 & $1.328(6)$ & N6-N7-C21 & $111.2(4)$ \\
N1-C2 & $1.353(5)$ & N1-C2-C3 & $108.3(4)$ \\
N3-C3 & $1.330(5)$ & N3-C3-C2 & $104.9(3)$ \\
N3-C4 & $1.456(5)$ & N4-C17-C12 & $119.9(4)$ \\
N4-C17 & $1.424(5)$ & N4-C18-C4 & $118.0(3)$ \\
N4-C18 & $1.358(5)$ & N4-C19-C20 & $112.1(3)$ \\
N4-C19 & $1.475(5)$ & N5-C20-C21 & $107.0(4)$ \\
N5-C20 & $1.348(6)$ & N7-C21-C20 & $105.4(4)$ \\
N7-C21 & $1.328(6)$ & C17-N4-C18 & $123.2(3)$ \\
N7-C22 & $1.481(6)$ & C19-N4-C17 & $121.9(4)$ \\
\hline
\end{tabular}

\section{Conclusions}

A collection of novel 1,2,3-triazole- and quinoline-2,4(1H,3H)-dione based bis-heterocycles functional derivatives was prepared and characterized by IR, NMR and HRMS. Appropriate starting compounds with 4-(acetoxymethyl)-1H-1,2,3-triazole moiety were firstly deacetylated, and the obtained corresponding alcohols were further oxidized to aldehydes and carboxylic acids. Investigation of transformation approaches was carried

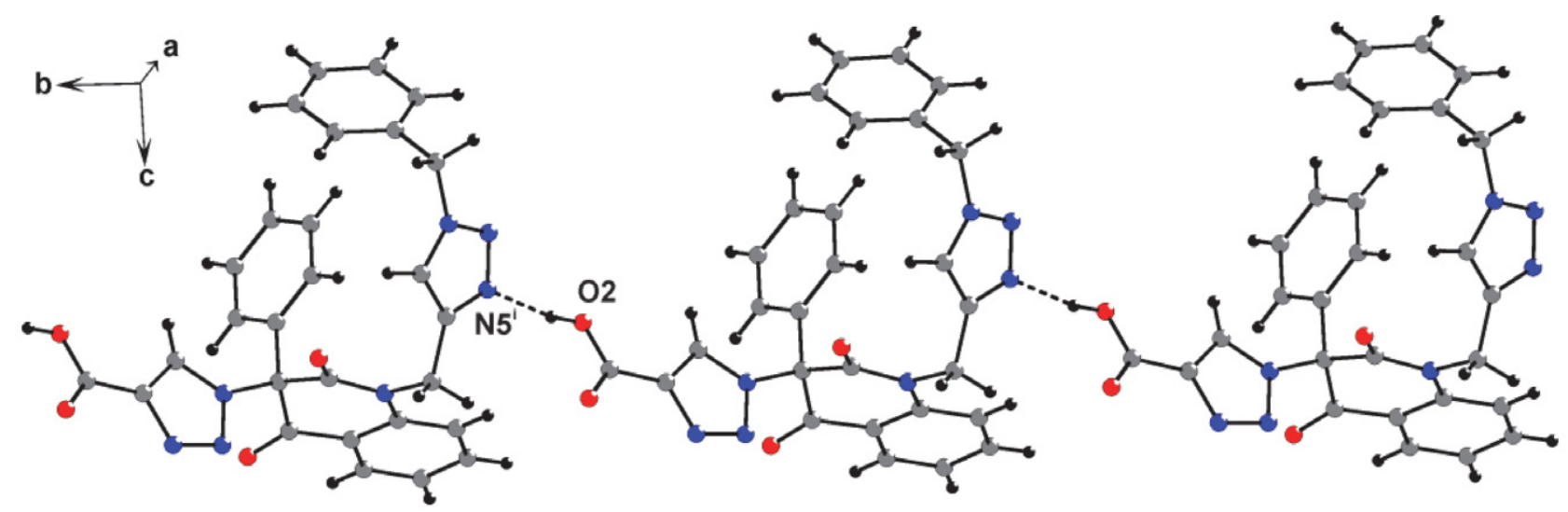

Figure 4. Hydrogen bonding interactions in the crystal structure of $\mathbf{1 2 d} \cdot 2 \mathrm{CDCl}_{3}$ showing the polymeric chain. Symmetry code: (i) $\mathrm{x}, \mathrm{y}+1, \mathrm{z}$.

Table 6. Hydrogen bonding geometry for compound $12 \mathrm{~d} \cdot 2 \mathrm{CDCl}_{3}$.

\begin{tabular}{|c|c|c|c|c|c|}
\hline $\mathrm{D}-\mathrm{H} \cdots \mathrm{A}$ & D-H $(\AA)$ & $\mathbf{H} \cdots \mathbf{A}(\AA)$ & $\mathbf{D} \cdots \mathbf{A}(\AA)$ & D-H $\cdots A\left(^{\circ}\right)$ & Symmetry code \\
\hline $\mathrm{O} 2-\mathrm{H} 2 \cdots \mathrm{N} 5$ & 0.82 & 1.90 & $2.700(5)$ & 166.7 & $\mathrm{x}, \mathrm{y}+1, \mathrm{z}$ \\
\hline
\end{tabular}


out using more accessible mono-triazoles, while optimized reaction conditions were then utilized for preparation of bis-triazole counterparts in moderate to excellent yields. Synthesized derivatives could potentially possess some desirable properties or might be exploited as precursors in further transformations.

In this article we present a group of new quinoline-2,4-dione based compounds with primary alcohol, aldehyde or carboxyl functional group on 1,2,3-triazole. Even though, the chemistry applied throughout the syntheses of our final materials is pretty elemental and straightforward, we believe that we have been handling with very promising substances and therefore, in our opinion, it was worthwhile to deal with them. Prepared compounds would not only potentially exhibit some extraordinary characteristics, but may also serve as precursors in further reactions such as esterification, peptide bond formation, nucleophilic additions to formyl group etc.

\section{Experimental}

The reagents and solvents were used as obtained from the commercial sources. Column chromatography was carried out on Fluka Silica gel 60 (particle size 0.063$0.2 \mathrm{~mm}$, activity acc. Brockmann and Schodder 2-3). Melting points were determined on the microscope hot stage, Kofler, PolyTherm, manufacturer Helmut Hund $\mathrm{GmbH}$, Wetzlar and are uncorrected. TLC was carried out on pre-coated TLC sheets ALUGRAM ${ }^{\circledR}$ SIL G/UV 254 for TLC, MACHEREY-NAGEL. NMR spectra were recorded with a Bruker Avance III $500 \mathrm{MHz}$ NMR instrument operating at $500 \mathrm{MHz}\left({ }^{1} \mathrm{H}\right), 126 \mathrm{MHz}\left({ }^{13} \mathrm{C}\right)$ and $51 \mathrm{MHz}\left({ }^{15} \mathrm{~N}\right)$ at $300 \mathrm{~K}$, or JEOL ECZ400R/S3 instrument operating at $400 \mathrm{MHz}\left({ }^{1} \mathrm{H}\right)$ and $100 \mathrm{MHz}\left({ }^{13} \mathrm{C}\right)$. Proton spectra were referenced to TMS as internal standard, in some cases to the residual signal of DMSO- $d_{5}$ (at $\delta 2.50 \mathrm{ppm}$ ) or $\mathrm{CHCl}_{3}$ (at $\delta 7.26 \mathrm{ppm}$ ). Carbon chemical shifts were determined relative to the ${ }^{13} \mathrm{C}$ signal of $\mathrm{DMSO}-d_{6}(39.52 \mathrm{ppm})$ or $\mathrm{CDCl}_{3}(77.16 \mathrm{ppm}) .{ }^{15} \mathrm{~N}$ chemical shifts were extracted from ${ }^{1} \mathrm{H}-{ }^{15} \mathrm{~N} g s$-HMBC spectra (with $20 \mathrm{~Hz}$ digital resolution in the indirect dimension and the parameters adjusted for a long-range ${ }^{1} \mathrm{H}-{ }^{15} \mathrm{~N}$ coupling constant of $5 \mathrm{~Hz}$ ) determined with respect to external nitromethane and are corrected to external ammonia by addition of $380.5 \mathrm{ppm}$. Nitrogen chemical shifts are reported to one decimal place as measured of the spectrum, however, the data should not be considered to be more accurate than $\pm 0.5 \mathrm{ppm}$ because of the digital resolution limits of the experiment. Chemical shifts are given on the $\delta$ scale (ppm). Coupling constants $(J)$ are given in $\mathrm{Hz}$. Multiplicities are indicated as follows: $\mathrm{s}$ (singlet), d (doublet), $\mathrm{t}$ (triplet), q (quartet), $\mathrm{m}$ (multiplet) or br (broadened). Infrared spectra were recorded on FTIR spectrometer Alpha (Bruker Optik GmbH Ettlingen, Germany) using samples in potassium bromide disks and only the strongest/structurally most important peaks are listed. HRMS spectra were recorded with Agilent 6224 Accurate Mass TOF LC/MS system with electrospray ionization (ESI).

$X$-ray crystallography. The molecular structure of compound 12d was determined by single-crystal X-ray diffraction methods. Crystallographic data and refinement details are given in Table 7. Diffraction data for 12d were collected at room temperature with Agilent SuperNova dual source diffractometer using an Atlas detector and equipped with mirror-monochromated $\mathrm{MoK} \alpha$ radiation $(\lambda=0.71073 \AA)$. The data were processed by using CrysAlis PRO.44 All the structures were solved using SHELXS-9745 and refined against $F^{2}$ on all data by full-matrix least-squares with SHELXL-2016. ${ }^{46}$ All non-hydrogen atoms were refined anisotropically. The C3 and $\mathrm{C} 21$ bonded hydrogen atoms were located in a difference map and refined with the distance restraints (DFIX) with $\mathrm{C}-\mathrm{H}=0.98 \AA$ and with $U_{\text {iso }}(\mathrm{H})=1.2 \mathrm{U}_{\mathrm{eq}}(\mathrm{C})$. All other hydrogen atoms were included in the model at geometrically calculated positions and refined using a riding model. The crystal structure 12d contains deuterated solvent molecules $\left(\mathrm{CDCl}_{3}\right)$. The $\mathrm{D}$ and $\mathrm{H}$ atoms are both treated as hydrogens but the SFAC instruction for D enables the formula weight and density to be calculated correctly. The C29 and C30 bonded deuterium atoms were located in a

Table 7. Crystal data and structure refinement details for compound $12 \mathrm{~d} \cdot 2 \mathrm{CDCl}_{3}$.

\begin{tabular}{|c|c|}
\hline & $12 \mathrm{~d} \cdot 2 \mathrm{CDCl}_{3}$ \\
\hline formula & $\mathrm{C}_{30} \mathrm{H}_{21} \mathrm{Cl}_{6} \mathrm{D}_{2} \mathrm{~N}_{7} \mathrm{O}_{4}$ \\
\hline $\mathrm{Fw}\left(\mathrm{g} \mathrm{mol}^{-1}\right)$ & 760.29 \\
\hline crystal size (mm) & $0.50 \times 0.30 \times 0.10$ \\
\hline crystal color & colourless \\
\hline crystal system & monoclinic \\
\hline space group & $P 2_{1} / n$ \\
\hline$a(\AA)$ & $13.5462(6)$ \\
\hline$b(\AA)$ & $11.9884(9)$ \\
\hline$c(\AA)$ & $20.8335(10)$ \\
\hline$\beta\left({ }^{\circ}\right)$ & $92.823(4)$ \\
\hline$V\left(\AA^{3}\right)$ & $3379.2(3)$ \\
\hline$Z$ & 4 \\
\hline calcd density $\left(\mathrm{g} \mathrm{cm}^{-3}\right)$ & 1.494 \\
\hline$F(000)$ & 1544 \\
\hline no. of collected reflns & 29191 \\
\hline no. of independent reflns & 7754 \\
\hline$R_{\text {int }}$ & 0.0563 \\
\hline no. of reflns observed & 3853 \\
\hline no. parameters & 438 \\
\hline$R[I>2 \sigma(I)]^{a}$ & 0.0974 \\
\hline$w R_{2}(\text { all data })^{b}$ & 0.3413 \\
\hline Goof,$S^{c}$ & 1.092 \\
\hline $\begin{array}{l}\text { maximum/minimum residual } \\
\text { electron density }\left(\mathrm{e} \AA^{-3}\right)\end{array}$ & $+0.90 /-0.80$ \\
\hline
\end{tabular}


difference map and refined with the distance restraints (DFIX) with C-D $=0.98 \AA$ and with $U_{\text {iso }}(\mathrm{D})=1.2 \mathrm{U}_{\mathrm{eq}}(\mathrm{C})$. CCDC 1892717 (for 12d $\cdot 2 \mathrm{CDCl}_{3}$ ) contain the supplementary crystallographic data for this paper. These data can be obtained free of charge from The Cambridge Crystallographic Data Centre via www.ccdc.cam.ac.uk/data request/cif.

General procedure for the synthesis of alcohols $5 a, b$ and 6a-f. A solution of appropriate acetate in acidified ethanol $(37 \% \mathrm{HCl}: \mathrm{EtOH}$ 1:100 V/V) was stirred at the reflux temperature $\left(90-100^{\circ} \mathrm{C}\right.$ in oil bath) for $2.5-4$ hours. Obtained pale yellow solution was then allowed to cool to room temperature, and subsequently neutralized with saturated aqueous $\mathrm{NaHCO}_{3}$. Resulting suspension was concentrated by rotary evaporation in vacuo, diluted with deionized water and extracted with chloroform (3-6x $50 \mathrm{~mL})$. Organic phases were joined together, washed with deionized water (1x50 mL), dried over anhydrous $\mathrm{Na}_{2} \mathrm{SO}_{4}$, filtered. and volatile components were evaporated in vacuo. The residual oily or solid product was then purified by chromatography on silica-gel column using 5\% ethanol or 30\% ethyl acetate in chloroform as eluent, or crystalized from ethyl acetate.

3-(4-(Hydroxymethyl)-1H-1,2,3-triazol-1-yl)-3-methyl1-(prop-2-ynyl)quinoline-2,4(1H,3H)-dione (5a). Colorless crystals, $\mathrm{mp} 182-188^{\circ} \mathrm{C}$ (ethyl acetate); $R_{\mathrm{f}}=0.12(5 \%$ ethanol in chloroform); $R_{\mathrm{f}}=0.31$ (10\% ethanol in chloroform); ${ }^{1} \mathrm{H}$ NMR (500 MHz, DMSO- $\left.d_{6}\right) \delta 2.08(\mathrm{~s}, 3 \mathrm{H})$, $3.35-3.38(\mathrm{~m}, 1 \mathrm{H}), 4.56(\mathrm{~d}, 2 \mathrm{H}, J=5.7 \mathrm{~Hz}), 4.84(\mathrm{dd}, 1 \mathrm{H}, J$ $=18.1,2.4 \mathrm{~Hz}), 4.95(\mathrm{dd}, 1 \mathrm{H}, J=18.1,2.4 \mathrm{~Hz}), 5.29(\mathrm{t}, 1 \mathrm{H}$, $J=5.7 \mathrm{~Hz}), 7.36(\mathrm{ddd}, 1 \mathrm{H}, J=7.6,7.4,0.9 \mathrm{~Hz}), 7.57(\mathrm{~d}, 1 \mathrm{H}$, $J=8.4 \mathrm{~Hz}), 7.89(\mathrm{ddd}, 1 \mathrm{H}, J=8.4,7.4,1.7 \mathrm{~Hz}), 7.96(\mathrm{dd}$, $1 \mathrm{H}, J=7.8,1.6 \mathrm{~Hz}), 8.26(\mathrm{~s}, 1 \mathrm{H}) ;{ }^{13} \mathrm{C} \mathrm{NMR}(126 \mathrm{MHz}$, DMSO- $\left.d_{6}\right) \delta 23.3,32.6,55.1,72.4,75.3,78.3,116.6,119.2$, $123.9,124.1,128.1,137.1,140.8,147.5,167.9,189.8$; IR $\left(\mathrm{cm}^{-1}\right): v 3270,3134,2126,1709,1677,1600,1465,1385$, 1301, 1180, 1022, 1011, 791, 762; HRMS (ESI+): $m / z$ calcd for $\mathrm{C}_{16} \mathrm{H}_{15} \mathrm{~N}_{4} \mathrm{O}_{3}{ }^{+}[\mathrm{M}+\mathrm{H}]^{+}$311.1139, found 311.1138.

3-(4-(Hydroxymethyl)-1H-1,2,3-triazol-1-yl)-3-phenyl1-(prop-2-ynyl)quinoline-2,4(1H,3H)-dione (5b). Colorless crystals, $\mathrm{mp} 141-148^{\circ} \mathrm{C}$ (ethyl acetate); $R_{\mathrm{f}}=0.21(5 \%$ ethanol in chloroform); $R_{\mathrm{f}}=0.46$ (10\% ethanol in chloroform); ${ }^{1} \mathrm{H} \mathrm{NMR}\left(500 \mathrm{MHz}, \mathrm{CDCl}_{3}\right) \delta 2.34$ (t, $1 \mathrm{H}, J=2.5$ $\mathrm{Hz}), 2.35-2.41(\mathrm{~m}, 1 \mathrm{H}), 4.48(\mathrm{dd}, 1 \mathrm{H}, J=17.8,2.4 \mathrm{~Hz})$, $4.71-4.79(\mathrm{~m}, 2 \mathrm{H}), 5.33(\mathrm{dd}, 1 \mathrm{H}, J=17.8,2.4 \mathrm{~Hz}), 7.05(\mathrm{~s}$, 1H), 7.22 (ddd, $1 \mathrm{H}, J=7.6,7.5,0.9 \mathrm{~Hz}), 7.33(\mathrm{~d}, 1 \mathrm{H}, J=8.3$ $\mathrm{Hz}$ ), 7.41-7.50 (m, 5H), 7.65 (ddd, $1 \mathrm{H}, J=8.3,7.4,1.7 \mathrm{~Hz}$ ), $8.03(\mathrm{dd}, 1 \mathrm{H}, J=7.8,1.6 \mathrm{~Hz}) ;{ }^{13} \mathrm{C} \mathrm{NMR}\left(126 \mathrm{MHz}, \mathrm{CDCl}_{3}\right)$ $\delta 33.6,56.8,73.6,79.7,115.8,120.9,124.6,128.9,129.2$, $129.7,130.1,131.3,136.9,140.5,145.8,165.7,187.5$; IR $\left(\mathrm{cm}^{-1}\right): v 3273,3158,2125,1715,1682,1602,1468,1374$, $1302,1175,1044,871,761$; HRMS (ESI + ): $\mathrm{m} / z$ calcd for $\mathrm{C}_{21} \mathrm{H}_{17} \mathrm{~N}_{4} \mathrm{O}_{3}{ }^{+}[\mathrm{M}+\mathrm{H}]^{+}$373.1295, found 373.1291.
1-((1-Benzyl-1H-1,2,3-triazol-4-yl)methyl)-3-(4-(hydroxymethyl)-1H-1,2,3-triazol-1-yl)-3-methylquinoline2,4(1H,3H)-dione (6a). Colorless powder, mp $69-98^{\circ} \mathrm{C}$; $R_{\mathrm{f}}=0.24$ (5\% ethanol in chloroform), $R_{\mathrm{f}}=0.11$ ( $3 \%$ ethanol in chloroform); ${ }^{1} \mathrm{H}$ NMR (500 MHz, $\left.\mathrm{CDCl}_{3}\right), \delta 2.11$ (s, $\left.3 \mathrm{H}, \mathrm{CH}_{3}\right), 2.59$ (s, $\left.1 \mathrm{H}, \mathrm{OH}\right), 4.80\left(\mathrm{~s}, 2 \mathrm{H}, \mathrm{OCH}_{2}\right), 5.29$ (d, $1 \mathrm{H}, J=15.8 \mathrm{~Hz}, \mathrm{~N}-1-\mathrm{CH} \alpha), 5.33(\mathrm{~d}, 1 \mathrm{H}, J=15.8 \mathrm{~Hz}$, $\mathrm{N}-1-\mathrm{CH} \beta), 5.44\left(\mathrm{~d}, 1 \mathrm{H}, J=14.8 \mathrm{~Hz}, \mathrm{~N}^{-}{ }^{\mathrm{C}}-\mathrm{CH} \alpha\right), 5.50(\mathrm{~d}$, $\left.1 \mathrm{H}, J=14.8 \mathrm{~Hz}, \mathrm{~N}-1^{\mathrm{C}}-\mathrm{CH} \beta\right), 7.19-7.28\left(\mathrm{~m}, 3 \mathrm{H}, \mathrm{H} 2^{\mathrm{D}}\right.$, H-6 $\left.{ }^{\mathrm{D}}, \mathrm{H}-6\right), 7.29-7.38\left(\mathrm{~m}, 3 \mathrm{H}, \mathrm{H}-3^{\mathrm{D}}, \mathrm{H}-4^{\mathrm{D}}, \mathrm{H}-5^{\mathrm{D}}\right), 7.56$ (s, $\left.1 \mathrm{H}, \mathrm{H}-5^{\mathrm{C}}\right), 7.67-7.74\left(\mathrm{~m}, 2 \mathrm{H}, \mathrm{H}-7, \mathrm{H}-5^{\mathrm{A}}\right), 7.79(\mathrm{~d}, 1 \mathrm{H}, J=$ $8.4 \mathrm{~Hz}, \mathrm{H}-8$ ), 7.99 (dd, $1 \mathrm{H}, J=7.8,1.6 \mathrm{~Hz}, \mathrm{H}-5) ;{ }^{13} \mathrm{C}$ NMR $\left(126 \mathrm{MHz}, \mathrm{CDCl}_{3}\right) \delta 23.5\left(\mathrm{CH}_{3}\right), 39.5\left(\mathrm{~N}-1-\mathrm{CH}_{2}\right), 54.5$ $\left(\mathrm{N}-1{ }^{\mathrm{C}}-\mathrm{CH}_{2}\right), 56.9\left(\mathrm{OCH}_{2}\right), 71.7(\mathrm{C}-3), 116.9(\mathrm{C}-8), 119.2$ (C-4a), $122.1\left(\mathrm{C}-5^{\mathrm{A}}\right), 123.5\left(\mathrm{C}-5^{\mathrm{C}}\right), 124.6(\mathrm{C}-6), 128.2(\mathrm{C}-$ $\left.2^{\mathrm{D}}, \mathrm{C}-6^{\mathrm{D}}\right), 129.0(\mathrm{C}-5), 129.3\left(\mathrm{C}-4^{\mathrm{D}}\right), 129.3\left(\mathrm{C}-3^{\mathrm{D}}, \mathrm{C}-5^{\mathrm{D}}\right)$, $134.4\left(\mathrm{C}-1^{\mathrm{D}}\right), 137.7(\mathrm{C}-7), 141.7(\mathrm{C}-8 \mathrm{a}), 142.9\left(\mathrm{C}-4^{\mathrm{C}}\right)$, $147.3\left(\mathrm{C}-4^{\mathrm{A}}\right), 168.3(\mathrm{C}-2), 189.6(\mathrm{C}-4) ;{ }^{15} \mathrm{~N}$ NMR $(51 \mathrm{MHz}$, $\left.\mathrm{CDCl}_{3}\right) \delta 138.6(\mathrm{~N}-1), 247.1\left(\mathrm{~N}-1^{\mathrm{A}}\right), 250.4\left(\mathrm{~N}-1^{\mathrm{C}}\right), 349.3$ $\left(\mathrm{N}-3^{\mathrm{C}}\right), 350.4\left(\mathrm{~N}-3^{\mathrm{A}}\right), 361.6\left(\mathrm{~N}-2^{\mathrm{C}}\right), 362.1\left(\mathrm{~N}-2^{\mathrm{A}}\right)$; IR $\left(\mathrm{cm}^{-1}\right): v 3413,3141,1714,1678,1602,1470,1384,1185$, 1051, 793, 762, 722, 664; HRMS (ESI+): $\mathrm{m} / z$ calcd for $\mathrm{C}_{23} \mathrm{H}_{22} \mathrm{~N}_{7} \mathrm{O}_{3}{ }^{+}[\mathrm{M}+\mathrm{H}]^{+} 444.1779$, found 444.1773 .

3-(4-(Hydroxymethyl)-1H-1,2,3-triazol-1-yl)-3-methyl1-((1-phenyl-1H-1,2,3-triazol-4-yl)methyl)quinoline-2,4(1H,3H)-dione (6b). Colorless powder, mp 96$115^{\circ} \mathrm{C} ; R_{\mathrm{f}}=0.41$ ( $10 \%$ ethanol in chloroform), $R_{\mathrm{f}}=0.17$ (5\% ethanol in chloroform); ${ }^{1} \mathrm{H}$ NMR $\left(500 \mathrm{MHz}, \mathrm{CDCl}_{3}\right)$ $\delta 2.17\left(\mathrm{~s}, 3 \mathrm{H}, \mathrm{CH}_{3}\right), 2.65(\mathrm{~s}, 1 \mathrm{H}, \mathrm{OH}), 4.82\left(\mathrm{~s}, 2 \mathrm{H}, \mathrm{OCH}_{2}\right)$, $5.37(\mathrm{~d}, 1 \mathrm{H}, J=15.8 \mathrm{~Hz}, \mathrm{~N}-1-\mathrm{CH} \alpha), 5.48(\mathrm{~d}, 1 \mathrm{H}, J=15.8$ $\mathrm{Hz}, \mathrm{N}-1-\mathrm{CH} \beta), 7.22-7.27$ (m, 1H, H-6), 7.39-7.44 (m, $1 \mathrm{H}$, $\left.\mathrm{H}-4^{\mathrm{D}}\right), 7.46-7.52\left(\mathrm{~m}, 2 \mathrm{H}, \mathrm{H}-3^{\mathrm{D}}, \mathrm{H}-5^{\mathrm{D}}\right), 7.68-7.75(\mathrm{~m}, 3 \mathrm{H}$, $\left.\mathrm{H}-2^{\mathrm{D}}, \mathrm{H}-6^{\mathrm{D}}, \mathrm{H}-7\right), 7.76\left(\mathrm{~s}, 1 \mathrm{H}, \mathrm{H}-5^{\mathrm{A}}\right), 7.82(\mathrm{~d}, 1 \mathrm{H}, J=8.4$ $\mathrm{Hz}, \mathrm{H}-8$ ), 8.01 (dd, $1 \mathrm{H}, J=7.8,1.6 \mathrm{~Hz}, \mathrm{H}-5$ ), 8.09 (s, $1 \mathrm{H}$, $\left.\mathrm{H}-5^{\mathrm{C}}\right) ;{ }^{13} \mathrm{C} \mathrm{NMR}\left(126 \mathrm{MHz}, \mathrm{CDCl}_{3}\right) \delta 23.4\left(\mathrm{CH}_{3}\right), 39.5$ $\left(\mathrm{N}-1-\mathrm{CH}_{2}\right), 56.9\left(\mathrm{OCH}_{2}\right), 71.6(\mathrm{C}-3), 116.8(\mathrm{C}-8), 119.2$ (C-4a), $120.6\left(\mathrm{C}-2^{\mathrm{D}}, \mathrm{C}^{-} \mathrm{6}^{\mathrm{D}}\right), 121.8\left(\mathrm{C}-5^{\mathrm{C}}\right), 122.1\left(\mathrm{C}-5^{\mathrm{A}}\right)$, $124.6(\mathrm{C}-6), 129.1\left(\mathrm{C}-4^{\mathrm{D}}\right), 129.4(\mathrm{C}-5), 129.9\left(\mathrm{C}-3^{\mathrm{D}}, \mathrm{C}-5^{\mathrm{D}}\right)$, $136.9\left(\mathrm{C}-1^{\mathrm{D}}\right), 137.8(\mathrm{C}-7), 141.7(\mathrm{C}-8 \mathrm{a}), 143.2\left(\mathrm{C}-4^{\mathrm{C}}\right)$, $147.3\left(\mathrm{C}-4^{\mathrm{A}}\right), 168.4(\mathrm{C}-2), 189.5(\mathrm{C}-4) ;{ }^{15} \mathrm{~N}$ NMR $(51 \mathrm{MHz}$, $\left.\mathrm{CDCl}_{3}\right) \delta 138.5(\mathrm{~N}-1), 247.4\left(\mathrm{~N}-1^{\mathrm{A}}\right), 256.2\left(\mathrm{~N}-1^{\mathrm{C}}\right), 350.8$ $\left(\mathrm{N}-3^{\mathrm{A}}\right)$, $351.6\left(\mathrm{~N}-3^{\mathrm{C}}\right)$; IR $\left(\mathrm{cm}^{-1}\right): v 3400,3143,1715,1678$, 1601, 1470, 1384, 1303, 1233, 1183, 1047, 760, 690, 663; HRMS (ESI+): $m / z$ calcd for $\mathrm{C}_{22} \mathrm{H}_{20} \mathrm{~N}_{7} \mathrm{O}_{3}{ }^{+}[\mathrm{M}+\mathrm{H}]^{+}$ 430.1622 , found 430.1614 .

3-(4-(Hydroxymethyl)-1H-1,2,3-triazol-1-yl)-3-methyl1-((1-(pyridin-2-yl)-1H-1,2,3-triazol-4-yl)methyl)quinoline-2,4(1H,3H)-dione (6c). Colorless powder, $\mathrm{mp}$ 66-89 ${ }^{\circ} \mathrm{C} ; R_{\mathrm{f}}=0.32$ (10\% ethanol in chloroform), $R_{\mathrm{f}}=0.09(5 \%$ ethanol in chloroform); ${ }^{1} \mathrm{H}$ NMR $\left(500 \mathrm{MHz}, \mathrm{CDCl}_{3}\right) \delta$ 2.19 (s, 3H, $\left.\mathrm{CH}_{3}\right), 2.32$ (s, $\left.1 \mathrm{H}, \mathrm{OH}\right), 4.83\left(\mathrm{~s}, 2 \mathrm{H}, \mathrm{OCH}_{2}\right)$, $5.36(\mathrm{~d}, 1 \mathrm{H}, J=15.9 \mathrm{~Hz}, \mathrm{~N}-1-\mathrm{CH} \alpha), 5.52(\mathrm{~d}, 1 \mathrm{H}, J=15.9$ $\mathrm{Hz}, \mathrm{N}-1-\mathrm{CH} \beta$ ), 7.23 (ddd, $1 \mathrm{H}, J=7.7,7.3,1.0 \mathrm{~Hz}, \mathrm{H}-6$ ), 7.30-7.37 (m, $\left.1 \mathrm{H}, \mathrm{H}-5^{\mathrm{D}}\right), 7.71(\mathrm{ddd}, 1 \mathrm{H}, J=8.5,7.2,1.6 \mathrm{~Hz}$, 
$\mathrm{H}-7)$, 7.75-7.78 (m, 2H, H-5 $\left.{ }^{\mathrm{A}}, \mathrm{H}-8\right), 7.87-7.92(\mathrm{~m}, 1 \mathrm{H}$, $\mathrm{H}-4^{\mathrm{D}}$ ), 8.01 (dd, $\left.1 \mathrm{H}, J=7.7,1.6 \mathrm{~Hz}, \mathrm{H}-5\right), 8.10-8.15$ (m, $\left.1 \mathrm{H}, \mathrm{H}-3^{\mathrm{D}}\right), 8.45-8.49\left(\mathrm{~m}, 1 \mathrm{H}, \mathrm{H}-6^{\mathrm{D}}\right), 8.58$ (s, $\left.1 \mathrm{H}, \mathrm{H}-5^{\mathrm{C}}\right)$; ${ }^{13} \mathrm{C}$ NMR $\left(126 \mathrm{MHz}, \mathrm{CDCl}_{3}\right) \quad \delta \quad 23.7 \quad\left(\mathrm{CH}_{3}\right), \quad 39.4$ $\left(\mathrm{N}-1-\mathrm{CH}_{2}\right), 56.9\left(\mathrm{OCH}_{2}\right), 72.0(\mathrm{C}-3), 113.9\left(\mathrm{C}-3^{\mathrm{D}}\right), 116.6$ $(\mathrm{C}-8), 119.3(\mathrm{C}-4 \mathrm{a}), 120.9\left(\mathrm{C}-5^{\mathrm{C}}\right), 122.3\left(\mathrm{C}-5^{\mathrm{A}}\right), 124.0$ $\left(\mathrm{C}-5^{\mathrm{D}}\right), 124.6$ (C-6), 129.3 (C-5), 137.6 (C-7), 139.3 $\left(\mathrm{C}-4^{\mathrm{D}}\right), 141.6(\mathrm{C}-8 \mathrm{a}), 143.0\left(\mathrm{C}-4^{\mathrm{C}}\right), 147.4\left(\mathrm{C}-4^{\mathrm{A}}\right), 148.8$ $\left(\mathrm{C}-6^{\mathrm{D}}\right), 149.0\left(\mathrm{C}-2^{\mathrm{D}}\right), 168.3(\mathrm{C}-2), 189.6(\mathrm{C}-4) ;{ }^{15} \mathrm{~N}$ NMR $\left(51 \mathrm{MHz}, \mathrm{CDCl}_{3}\right) \delta 137.7(\mathrm{~N}-1), 246.7\left(\mathrm{~N}-1^{\mathrm{A}}\right), 259.9$ $\left(\mathrm{N}-1^{\mathrm{C}}\right), 283.6\left(\mathrm{~N}-1^{\mathrm{D}}\right), 350.3\left(\mathrm{~N}-3^{\mathrm{A}}\right), 355.0\left(\mathrm{~N}-3^{\mathrm{C}}\right)$; IR $\left(\mathrm{cm}^{-1}\right): v 3379,3132,1715,1679,1600,1471,1384,1298$, 1234, 1183, 1040, 782, 755, 658; HRMS (ESI+): $m / z$ calcd for $\mathrm{C}_{21} \mathrm{H}_{19} \mathrm{~N}_{8} \mathrm{O}_{3}{ }^{+}[\mathrm{M}+\mathrm{H}]^{+} 431.1575$, found 431.1579.

1-((1-Benzyl-1H-1,2,3-triazol-4-yl)methyl)-3-(4-(hydroxymethyl)-1H-1,2,3-triazol-1-yl)-3-phenylquinoline2,4(1H,3H)-dione (6d). Colorless powder, mp 93-121 ${ }^{\circ} \mathrm{C}$; $R_{\mathrm{f}}=0.23$ (5\% ethanol in chloroform); ${ }^{1} \mathrm{H}$ NMR $(500 \mathrm{MHz}$, $\left.\mathrm{CDCl}_{3}\right) \delta 2.34(\mathrm{t}, 1 \mathrm{H}, J=5.6 \mathrm{~Hz}, \mathrm{OH}), 4.75(\mathrm{~d}, 2 \mathrm{H}, J=$ $\left.4.6 \mathrm{~Hz}, \mathrm{OCH}_{2}\right), 5.20\left(\mathrm{~d}, 1 \mathrm{H}, J=15.6 \mathrm{~Hz}, \mathrm{~N}-1^{\mathrm{C}}-\mathrm{CH} \alpha\right), 5.43$ $(\mathrm{d}, 1 \mathrm{H}, J=14.8 \mathrm{~Hz}, \mathrm{~N}-1-\mathrm{CH} \alpha), 5.49(\mathrm{~d}, 1 \mathrm{H}, J=15.6 \mathrm{~Hz}$, $\left.\mathrm{N}-1^{\mathrm{C}}-\mathrm{CH} \beta\right), 5.54(\mathrm{~d}, 1 \mathrm{H}, J=14.8 \mathrm{~Hz}, \mathrm{~N}-1-\mathrm{CH} \beta), 7.03$ (s, $1 \mathrm{H}, \mathrm{H} 5^{\mathrm{A}}$ ), 7.17 (ddd, $\left.1 \mathrm{H}, J=7.9,7.2,0.8 \mathrm{~Hz}, \mathrm{H}-6\right), 7.23-$ $7.28\left(\mathrm{~m}, 4 \mathrm{H}, \mathrm{H}-2^{\mathrm{D}}, \mathrm{H}-3^{\mathrm{D}}, \mathrm{H}-5^{\mathrm{D}}, \mathrm{H}-6^{\mathrm{D}}\right), 7.29-7.32(\mathrm{~m}, 2 \mathrm{H}$, $\left.\mathrm{H}-2^{\mathrm{B}}, \mathrm{H}-6^{\mathrm{B}}\right), 7.34-7.42\left(\mathrm{~m}, 4 \mathrm{H}, \mathrm{H}-3^{\mathrm{B}}, \mathrm{H}-4^{\mathrm{B}}, \mathrm{H}-5^{\mathrm{B}}, \mathrm{H}-4^{\mathrm{D}}\right)$, $7.59\left(\mathrm{~s}, 1 \mathrm{H}, \mathrm{H} 5^{\mathrm{C}}\right), 7.62(\mathrm{ddd}, 1 \mathrm{H}, J=7.9,7.9,1.6 \mathrm{~Hz}, \mathrm{H}-7)$, $7.74(\mathrm{~d}, 1 \mathrm{H}, J=8.4 \mathrm{~Hz}, \mathrm{H}-8), 7.98(\mathrm{dd}, 1 \mathrm{H}, J=7.7,1.6 \mathrm{~Hz}$, $\mathrm{H}-5) ;{ }^{13} \mathrm{C} \mathrm{NMR}\left(126 \mathrm{MHz}, \mathrm{CDCl}_{3}\right) \delta{ }^{13} \mathrm{C} \mathrm{NMR}(126 \mathrm{MHz}$, $\left.\mathrm{CDCl}_{3}\right) \delta 39.9\left(\mathrm{~N}-1^{\mathrm{C}}-\mathrm{CH}_{2}\right), 54.5\left(\mathrm{~N}-1-\mathrm{CH}_{2}\right), 56.9\left(\mathrm{OCH}_{2}\right)$, 79.6 (C-3), 116.8 (C-8), 120.9 (C-4a), $123.6\left(\mathrm{C}-5^{\mathrm{C}}\right), 124.5$ $\left(\mathrm{C}-5^{\mathrm{A}}\right), 124.5(\mathrm{C}-6), 128.3\left(\mathrm{C}-2^{\mathrm{D}}, \mathrm{C}-6^{\mathrm{D}}\right), 128.8\left(\mathrm{C}-2^{\mathrm{B}}\right.$, C-6 $\left.{ }^{\mathrm{B}}\right), 129.0(\mathrm{C}-5), 129.0\left(\mathrm{C}-4^{\mathrm{B}}\right), 129.3\left(\mathrm{C}-3^{\mathrm{B}}, \mathrm{C}-5^{\mathrm{B}}\right), 129.9$ $\left(\mathrm{C}-1^{\mathrm{D}}\right), 130.0\left(\mathrm{C}-3^{\mathrm{D}}, \mathrm{C}-5^{\mathrm{D}}\right), 131.2\left(\mathrm{C}-4^{\mathrm{D}}\right), 134.5\left(\mathrm{C}-1^{\mathrm{B}}\right)$, 137.2 (C-7), 141.1 (C-8a), $142.9\left(\mathrm{C}-4^{\mathrm{C}}\right), 145.8\left(\mathrm{C}-4^{\mathrm{A}}\right)$, 166.6 (C-2), 188.0 (C-4); ${ }^{15} \mathrm{~N}$ NMR (51 MHz, $\left.\mathrm{CDCl}_{3}\right) \delta$ $140.4(\mathrm{~N}-1), 248.9\left(\mathrm{~N}-1^{\mathrm{A}}\right), 250.6\left(\mathrm{~N}-1^{\mathrm{C}}\right), 350.0\left(\mathrm{~N}-3^{\mathrm{C}}\right)$, $352.8\left(\mathrm{~N}-3^{\mathrm{A}}\right), 362.8\left(\mathrm{~N}-2^{\mathrm{C}}\right), 364.9\left(\mathrm{~N}-2^{\mathrm{A}}\right)$; IR $\left(\mathrm{cm}^{-1}\right): v$ $3391,3141,1715,1678,1601,1469,1450,1376,1049,1032$, 871, 761, 665, 608; HRMS (ESI+): $\mathrm{m} / z$ calcd for $\mathrm{C}_{28} \mathrm{H}_{24} \mathrm{~N}_{7} \mathrm{O}_{3}{ }^{+}[\mathrm{M}+\mathrm{H}]^{+}$506.1935, found 506.1937.

3-(4-(Hydroxymethyl)-1H-1,2,3-triazol-1-yl)-3-phenyl1-((1-phenyl-1H-1,2,3-triazol-4-yl)methyl)quinoline-2,4(1H,3H)-dione (6e). Colorless powder, mp 118$131{ }^{\circ} \mathrm{C} ; R_{\mathrm{f}}=0.35$ (5\% ethanol in chloroform), $R_{\mathrm{f}}=0.22(3 \%$ ethanol in chloroform); ${ }^{1} \mathrm{H}$ NMR $\left(500 \mathrm{MHz}, \mathrm{CDCl}_{3}\right) \delta$ $2.33(\mathrm{~s}, 1 \mathrm{H}, \mathrm{OH}), 4.78\left(\mathrm{~s}, 2 \mathrm{H}, \mathrm{OCH}_{2}\right), 5.41(\mathrm{~d}, 1 \mathrm{H}, J=15.7$ $\mathrm{Hz}, \mathrm{N}-1-\mathrm{CH} \alpha$ ), 5.53 (d, $1 \mathrm{H}, J=15.7 \mathrm{~Hz}, \mathrm{~N}-1-\mathrm{CH} \beta$ ), 7.09 $\left(\mathrm{s}, 1 \mathrm{H}, \mathrm{H}-5^{\mathrm{A}}\right), 7.20$ (ddd, $\left.1 \mathrm{H}, J=7.9,7.2,0.7 \mathrm{~Hz}, \mathrm{H}-6\right)$, 7.38-7.48 (m, 6H, H-2 $\left.2^{\mathrm{B}}, \mathrm{H}-3^{\mathrm{B}}, \mathrm{H}-4^{\mathrm{B}}, \mathrm{H}-5^{\mathrm{B}}, \mathrm{H}-6^{\mathrm{B}}, \mathrm{H}-4^{\mathrm{D}}\right)$, 7.49-7.55 (m, 2H, H-3 $\left.{ }^{\mathrm{D}}, \mathrm{H}-5^{\mathrm{D}}\right), 7.65$ (ddd, $1 \mathrm{H}, J=8.7,7.1$, $1.7 \mathrm{~Hz}, \mathrm{H}-7), 7.68-7.72\left(\mathrm{~m}, 2 \mathrm{H}, \mathrm{H} 2^{\mathrm{D}}, \mathrm{H}-6^{\mathrm{D}}\right), 7.75$ (d, $1 \mathrm{H}$, $J=8.4 \mathrm{~Hz}, \mathrm{H}-8), 8.02(\mathrm{dd}, 1 \mathrm{H}, J=7.8,1.5 \mathrm{~Hz}, \mathrm{H}-5), 8.07$ (s, $\left.1 \mathrm{H}, \mathrm{H} 5{ }^{\mathrm{C}}\right) ;{ }^{13} \mathrm{C} \mathrm{NMR}\left(126 \mathrm{MHz}, \mathrm{CDCl}_{3}\right) \delta 39.8\left(\mathrm{~N}-1-\mathrm{CH}_{2}\right)$, $56.9\left(\mathrm{OCH}_{2}\right), 79.6(\mathrm{C}-3), 116.7(\mathrm{C}-8), 120.7\left(\mathrm{C}-2^{\mathrm{D}}, \mathrm{C}-6^{\mathrm{D}}\right)$,
$120.9(\mathrm{C}-4 \mathrm{a}), 121.8\left(\mathrm{C}-5^{\mathrm{C}}\right), 124.6\left(\mathrm{C}-5^{\mathrm{A}}\right), 124.6(\mathrm{C}-6)$, $128.9\left(\mathrm{C}-2^{\mathrm{B}}, \mathrm{C}-6^{\mathrm{B}}\right), 129.1(\mathrm{C}-5), 129.2\left(\mathrm{C}-4^{\mathrm{D}}\right), 130.0$ $\left(\mathrm{C}-1^{\mathrm{B}}\right), 130.0\left(\mathrm{C}-3^{\mathrm{D}}, \mathrm{C}-5^{\mathrm{D}}\right), 130.1\left(\mathrm{C}-3^{\mathrm{B}}, \mathrm{C}-5^{\mathrm{B}}\right), 131.3$ $\left(\mathrm{C}-4^{\mathrm{B}}\right), 136.9\left(\mathrm{C}-1^{\mathrm{D}}\right), 137.3(\mathrm{C}-7), 140.9(\mathrm{C}-8 \mathrm{a}), 143.2(\mathrm{C}-$ $\left.4^{\mathrm{C}}\right), 145.8\left(\mathrm{C}-4^{\mathrm{A}}\right), 166.9(\mathrm{C}-2), 188.0(\mathrm{C}-4) ;{ }^{15} \mathrm{~N}$ NMR $(51$ $\left.\mathrm{MHz}, \mathrm{CDCl}_{3}\right) \delta 139.5(\mathrm{~N}-1), 249.0\left(\mathrm{~N}-1^{\mathrm{A}}\right), 256.2\left(\mathrm{~N}-1^{\mathrm{C}}\right)$, $352.6\left(\mathrm{~N}-3^{\mathrm{C}}\right), 353.0\left(\mathrm{~N}-3^{\mathrm{A}}\right)$; IR $\left(\mathrm{cm}^{-1}\right): v$ 3401, 3144, 1716, 1679, 1600, 1501, 1468, 1449, 1377, 1044, 871, 760, 692; HRMS (ESI+): $m / z$ calcd for $\mathrm{C}_{27} \mathrm{H}_{22} \mathrm{~N}_{7} \mathrm{O}_{3}{ }^{+}[\mathrm{M}+\mathrm{H}]^{+}$ 492.1779, found 492.1768 .

3-(4-(Hydroxymethyl)-1H-1,2,3-triazol-1-yl)-3-phenyl1-((1-(pyridin-2-yl)-1H-1,2,3-triazol-4-yl)methyl)quinoline-2,4(1H,3H)-dione (6f). Colorless powder, mp 185$194{ }^{\circ} \mathrm{C} ; R_{\mathrm{f}}=0.37$ (5\% ethanol in chloroform); ${ }^{1} \mathrm{H}$ NMR $\left(400 \mathrm{MHz}, \mathrm{CDCl}_{3}\right) \delta 2.38(\mathrm{~s}, 1 \mathrm{H}, \mathrm{OH}), 4.77\left(\mathrm{~s}, 2 \mathrm{H}, \mathrm{OCH}_{2}\right)$, $5.28(\mathrm{~d}, 1 \mathrm{H}, J=15.8 \mathrm{~Hz}, \mathrm{~N}-1-\mathrm{CH} \alpha), 5.69(\mathrm{~d}, 1 \mathrm{H}, J=15.8$ $\mathrm{Hz}, \mathrm{N}-1-\mathrm{CH} \beta$ ), 7.09 (s, $\left.1 \mathrm{H}, \mathrm{H}-5^{\mathrm{A}}\right), 7.17$ (ddd, $1 \mathrm{H}, J=7.7$, 7.3, $1.0 \mathrm{~Hz}, \mathrm{H}-6), 7.32-7.48\left(\mathrm{~m}, 6 \mathrm{H}, \mathrm{H}-5^{\mathrm{D}}, \mathrm{H}-2^{\mathrm{B}}, \mathrm{H}-3^{\mathrm{B}}\right.$, $\mathrm{H}-4^{\mathrm{B}}, \mathrm{H}-5^{\mathrm{B}}, \mathrm{H}-6^{\mathrm{B}}$ ), 7.61 (ddd, $1 \mathrm{H}, J=8.4,7.2,1.7 \mathrm{~Hz}, \mathrm{H}-7$ ), $7.68(\mathrm{~d}, 1 \mathrm{H}, J=8.3 \mathrm{~Hz}, \mathrm{H}-8), 7.86-7.94\left(\mathrm{~m}, 1 \mathrm{H}, \mathrm{H}-4^{\mathrm{D}}\right)$, $8.01(\mathrm{dd}, 1 \mathrm{H}, J=7.8,1.5 \mathrm{~Hz}, \mathrm{H}-5), 8.11-8.16(\mathrm{~m}, 1 \mathrm{H}$, H-3 $\left.{ }^{\mathrm{D}}\right), 8.47-8.51$ (m, $\left.1 \mathrm{H}, \mathrm{H}-6^{\mathrm{D}}\right), 8.61\left(\mathrm{~s}, 1 \mathrm{H}, \mathrm{H}-5^{\mathrm{C}}\right) ;{ }^{13} \mathrm{C}$ NMR $\left(100 \mathrm{MHz}, \mathrm{CDCl}_{3}\right) \delta 39.9\left(\mathrm{~N}-1-\mathrm{CH}_{2}\right), 56.9\left(\mathrm{OCH}_{2}\right)$, 79.7 (C-3), $113.9\left(\mathrm{C}-3^{\mathrm{D}}\right), 116.6(\mathrm{C}-8), 121.0(\mathrm{C}-4 \mathrm{a}), 121.0$ $\left(\mathrm{C}-5^{\mathrm{C}}\right), 124.0\left(\mathrm{C}-5^{\mathrm{D}}\right), 124.5(\mathrm{C}-6), 124.5\left(\mathrm{C}-5^{\mathrm{A}}\right), 128.9(\mathrm{C}-$ $\left.2^{\mathrm{B}}, \mathrm{C}-6^{\mathrm{B}}\right), 129.0(\mathrm{C}-5), 130.0\left(\mathrm{C}-1^{\mathrm{B}}\right), 130.1\left(\mathrm{C}-3^{\mathrm{B}}, \mathrm{C}-5^{\mathrm{B}}\right)$, $131.2\left(\mathrm{C}-4^{\mathrm{B}}\right), 137.1(\mathrm{C}-7), 139.3\left(\mathrm{C}-4^{\mathrm{D}}\right), 141.2(\mathrm{C}-8 \mathrm{a})$, $143.0\left(\mathrm{C}-4^{\mathrm{C}}\right), 145.9\left(\mathrm{C}-4^{\mathrm{A}}\right), 148.9\left(\mathrm{C}-6^{\mathrm{D}}\right), 149.0\left(\mathrm{C}-2^{\mathrm{D}}\right)$, 166.7 (C-2), $188.0(\mathrm{C}-4)$; IR $\left(\mathrm{cm}^{-1}\right): v$ 3401, 3156, 1716, 1680, 1599, 1469, 1375, 1313, 1034, 999, 779, 760, 695, 683; HRMS (ESI+): $m / z$ calcd for $\mathrm{C}_{26} \mathrm{H}_{21} \mathrm{~N}_{8} \mathrm{O}_{3}{ }^{+}[\mathrm{M}+\mathrm{H}]^{+}$ 493.1731, found 493.1732 .

General procedure for the preparation of aldehydes $7 \mathrm{a}, \mathrm{b}, 8 \mathrm{a}, \mathrm{b}$ and $9 \mathrm{a}-\mathrm{f}$ using $\mathrm{PCC}$ as the reagent. To a vigorously stirred solution of suitable alcohol $(1 \mathrm{mmol})$ in dichloromethane or acetone $(15 \mathrm{~mL})$, PCC (259 mg; 1.2 mmol) was added and the reaction mixture was stirred at the reflux temperature unless otherwise stated. Obtained reaction mixture was then stirred at the reflux temperature for up to one hour. The original orange color of mixture changed to almost black. Resulting solution with the sticky sediment was poured into a narrow $(1 \mathrm{~cm}$ in diameter) column of silica gel (13 g). The organic portion was eluted with $5 \%$ ethanol in chloroform (approximately $350 \mathrm{~mL}$ ). Volatile components of dark yellow eluate were evaporated in vacuo and obtained residue was chromatographed on a column of silica-gel (35 g) using $50 \%$ or $67 \%$ ethyl acetate in petroleum ether. Some crude products were further crystalized from ethyl acetate or benzene. For the reaction conditions and yields see Table 2 or Table 3, respectively.

General procedure for the preparation of aldehydes 7a,b using Swern reaction. To a dry $25 \mathrm{~mL}$ evacuated flask, oxalyl chloride $(155 \mu \mathrm{L} ; 1.8 \mathrm{mmol})$ and dry tetrahydrofurane 
(THF) were added. The flask was equipped with nitrogen gas inlet and cooled to $-70^{\circ} \mathrm{C}$ using dry ice-ethanol bath. Afterwards, DMSO $(280 \mu \mathrm{L})$ was added dropwise and obtained solution was stirred for 60 minutes, keeping the temperature bellow $-65^{\circ} \mathrm{C}$. Then, suitable mono-triazole alcohol $4(1.5 \mathrm{mmol})$ dissolved in dry dichloromethane or acetone $(11 \mathrm{~mL})$ was added and stirring was continued for 90 minutes. Finally, after addition of DIPEA $(1.275 \mathrm{~mL}$; $7.32 \mathrm{mmol}$ ), the content of the flask was stirred for additional 2 hours and tempered to the lab temperature. The reaction mixture was diluted with distilled water $(10 \mathrm{~mL})$ and extracted with dichloromethane (3x $20 \mathrm{~mL})$. Combined organic phases were washed with ice-cold water $(4 \mathrm{x}$ $20 \mathrm{~mL}$ ), dried over anhydrous $\mathrm{Na}_{2} \mathrm{SO}_{4}$, filtered and volatile components were evaporated in vacuo. Obtained oily crude product was purified on silica-gel column, using $38 \%$ ethyl acetate in petroleum ether as mobile phase. To that way gained oily product, diethyl ether was added and it was cooled to $-20{ }^{\circ} \mathrm{C}$ to provide solid compound that was filtered through the sintered glass filter and dried at 50 ${ }^{\circ} \mathrm{C}$. For the yields of products see Table 2 .

General procedure for the synthesis of aldehydes using $\mathrm{MnO}_{2}$ as a reagent. To a vigorously stirred solution of suitable alcohol $(1 \mathrm{mmol})$ in acetone $(10 \mathrm{~mL}), \mathrm{MnO}_{2}(869$ $\mathrm{mg} ; 10 \mathrm{mmol}$ ) was added. Obtained reaction mixture was then stirred at the reflux temperature unless otherwise stated. Resulting black suspension was filtered through the filter paper and volatile components of the filtrate were evaporated in vacuo. Residual crude oily product was chromatographed on silica-gel column, using 50\% ethyl acetate in petroleum ether as mobile phase. Some that way obtained TLC and IR pure products were further crystalized from ethyl acetate. For the reaction conditions and yields see Table 2 or Table 3 , respectively.

1-(1,2,3,4-Tetrahydro-3-methyl-2,4-dioxoquinolin-3-yl)-1H-1,2,3-triazole-4-carbaldehyde (7a). Colorless crystals, $\mathrm{mp} 267-271{ }^{\circ} \mathrm{C}$ (ethyl acetate); $R_{\mathrm{f}}=0.54(10 \%$ ethanol in chloroform); ${ }^{1} \mathrm{H}$ NMR $\left(500 \mathrm{MHz}, \mathrm{DMSO}-d_{6}\right) \delta$ $2.16\left(\mathrm{~s}, 3 \mathrm{H}, \mathrm{CH}_{3}\right), 7.23$ (d, $\left.1 \mathrm{H}, J=7.7 \mathrm{~Hz}, \mathrm{H}-8\right), 7.21-7.27$ (m, $1 \mathrm{H}, \mathrm{H}-6$ ), 7.75 (ddd, $1 \mathrm{H}, J=7.8,7.7,1.5 \mathrm{~Hz}, \mathrm{H}-7$ ), 7.85 (dd, $1 \mathrm{H}, J=7.8,1.7 \mathrm{~Hz}, \mathrm{H}-5), 9.18\left(\mathrm{~s}, 1 \mathrm{H}, \mathrm{H}-5^{\mathrm{A}}\right), 10.08$ (s, $1 \mathrm{H}, \mathrm{CHO}), 11.50(\mathrm{~s}, 1 \mathrm{H}, \mathrm{H}-1) ;{ }^{13} \mathrm{C}$ NMR $(126 \mathrm{MHz}$, DMSO- $\left.d_{6}\right) \delta 23.4\left(\mathrm{CH}_{3}\right), 73.6(\mathrm{C}-3), 117.0(\mathrm{C}-8), 117.6(\mathrm{C}-$ 4a), 123.5 (C-6), $127.6(\mathrm{C}-5), 129.6\left(\mathrm{C}-5^{\mathrm{A}}\right), 137.2(\mathrm{C}-7)$, $141.4(\mathrm{C}-8 \mathrm{a}), 146.6\left(\mathrm{C}-4^{\mathrm{A}}\right), 168.3(\mathrm{C}-2), 185.1(\mathrm{CHO})$, $190.3(\mathrm{C}-4) ;{ }^{15} \mathrm{~N}$ NMR $\left(51 \mathrm{MHz}, \mathrm{DMSO}-d_{6}\right) \delta 133.4(\mathrm{~N}-1)$, $252.0\left(\mathrm{~N}-1^{\mathrm{A}}\right), 358.6\left(\mathrm{~N}-3^{\mathrm{A}}\right), 367.9\left(\mathrm{~N}-2^{\mathrm{A}}\right)$; IR $\left(\mathrm{cm}^{-1}\right): v$ 3308, 3140, 2851, 1716, 1680, 1614, 1531, 1484, 1378, 1345, 1231, 1211, 816, 757, 667; HRMS (ESI+): $\mathrm{m} / z$ calcd for $\mathrm{C}_{13} \mathrm{H}_{11} \mathrm{~N}_{4} \mathrm{O}_{3}{ }^{+}[\mathrm{M}+\mathrm{H}]^{+}$271.0826, found 271.0833.

1-(1,2,3,4-Tetrahydro-2,4-dioxo-3-phenylquinolin-3-yl)1H-1,2,3-triazole-4-carbaldehyde (7b). Colorless crystals, mp $188-191{ }^{\circ} \mathrm{C}$ (ethyl acetate); $R_{\mathrm{f}}=0.36$ (5\% ethanol in chloroform), $R_{\mathrm{f}}=0.35$ (30\% ethyl acetate in chloroform); ${ }^{1} \mathrm{H}$ NMR (500 MHz, DMSO- $\left.d_{6}\right) \delta 7.09(\mathrm{~d}, 1 \mathrm{H}, J=$ $8.0 \mathrm{~Hz}, \mathrm{H}-8$ ), 7.17 (ddd, $1 \mathrm{H}, J=7.6,7.6,1.0 \mathrm{~Hz}, \mathrm{H}-6$ ), 7.34-7.41 (m, 2H, H-2 $\left.2^{\mathrm{B}}, \mathrm{H}-6^{\mathrm{B}}\right), 7.47-7.54\left(\mathrm{~m}, 3 \mathrm{H}, \mathrm{H}-3^{\mathrm{B}}\right.$, $\left.\mathrm{H}-4^{\mathrm{B}}, \mathrm{H}-5^{\mathrm{B}}\right), 7.63(\mathrm{ddd}, 1 \mathrm{H}, J=8.2,7.3,1.6 \mathrm{~Hz}, \mathrm{H}-7), 7.85$ (dd, $1 \mathrm{H}, J=7.8,1.4 \mathrm{~Hz}, \mathrm{H}-5), 8.93\left(\mathrm{~s}, 1 \mathrm{H}, \mathrm{H}-5^{\mathrm{A}}\right), 10.05$ (s, $1 \mathrm{H}, \mathrm{CHO}), 11.68(\mathrm{~s}, 1 \mathrm{H}, \mathrm{H}-1) ;{ }^{13} \mathrm{C}$ NMR $(126 \mathrm{MHz}$, DMSO- $\left.d_{6}\right) \delta 80.7$ (C-3), 116.7 (C-8), 119.5 (C-4a), 123.5 (C-6), $127.5(\mathrm{C}-5), 128.9\left(\mathrm{C}-2^{\mathrm{B}}, \mathrm{C}-6^{\mathrm{B}}\right), 129.7\left(\mathrm{C}-3^{\mathrm{B}}, \mathrm{C}-5^{\mathrm{B}}\right)$, $129.7\left(\mathrm{C}-1^{\mathrm{B}}\right), 130.7\left(\mathrm{C}-4^{\mathrm{B}}\right), 130.7\left(\mathrm{C}-5^{\mathrm{A}}\right), 136.8(\mathrm{C}-7)$, $140.4(\mathrm{C}-8 \mathrm{a}), 146.2\left(\mathrm{C}-4^{\mathrm{A}}\right), 166.6(\mathrm{C}-2), 185.1(\mathrm{CHO})$, $188.4(\mathrm{C}-4) ;{ }^{15} \mathrm{~N}$ NMR $\left(51 \mathrm{MHz}, \mathrm{DMSO}-d_{6}\right) \delta 134.8(\mathrm{~N}-1)$, $249.8\left(\mathrm{~N}-1^{\mathrm{A}}\right), 351.6\left(\mathrm{~N}-3^{\mathrm{A}}\right), 356.4\left(\mathrm{~N}-2^{\mathrm{A}}\right)$; IR $\left(\mathrm{cm}^{-1}\right): v$ $3253,2914,2860,1723,1689,1615,1595,1486,1355,1208$, 1045, 857, 780, 752, 697; HRMS (ESI+): $\mathrm{m} / z$ calcd for $\mathrm{C}_{18} \mathrm{H}_{13} \mathrm{~N}_{4} \mathrm{O}_{3}{ }^{+}[\mathrm{M}+\mathrm{H}]^{+}$333.0982, found 333.0988.

1-(1,2,3,4-Tetrahydro-3-methyl-2,4-dioxo-1-(prop-2ynyl)quinolin-3-yl)-1H-1,2,3-triazole-4-carbaldehyde (8a). Colorless crystals, mp $189-194{ }^{\circ} \mathrm{C}$ (benzene); $R_{\mathrm{f}}=0.40$ ( $5 \%$ ethanol in chloroform); $R_{\mathrm{f}}=0.63$ ( $10 \%$ ethanol in chloroform); ${ }^{1} \mathrm{H}$ NMR (500 MHz, $\left.\mathrm{CDCl}_{3}\right) \delta 2.23$ (s, $3 \mathrm{H}), 3.32-3.37(\mathrm{~m}, 1 \mathrm{H}), 4.66(\mathrm{dd}, 1 \mathrm{H}, J=17.9,1.6 \mathrm{~Hz})$, $5.11(\mathrm{dd}, 1 \mathrm{H}, J=17.9,1.6 \mathrm{~Hz}), 7.30-7.37(\mathrm{~m}, 1 \mathrm{H}), 7.47(\mathrm{~d}$, $1 \mathrm{H}, J=8.4 \mathrm{~Hz}$ ), 7.81 (ddd, $1 \mathrm{H}, J=8.4,7.3,1.5 \mathrm{~Hz}), 8.09$ (d, $1 \mathrm{H}, J=7.7 \mathrm{~Hz}), 8.31(\mathrm{~s}, 1 \mathrm{H}), 10.18(\mathrm{~s}, 1 \mathrm{H}),{ }^{13} \mathrm{C}$ NMR $(126$ $\left.\mathrm{MHz}, \mathrm{CDCl}_{3}\right) \delta 23.9,33.2,72.8,73.9,76.7,116.3,119.2$, 124.9, 126.3, 129.6, 137.7, 140.9, 147.1, 166.9, 185.1, 188.7; IR $\left(\mathrm{cm}^{-1}\right): v 3282,3150,2125,1704,1673,1601,1528$, 1470, 1444, 1381, 1306, 1206, 798, 761; HRMS (ESI+): $\mathrm{m} / \mathrm{z}$ calcd for $\mathrm{C}_{16} \mathrm{H}_{13} \mathrm{~N}_{4} \mathrm{O}_{3}{ }^{+}[\mathrm{M}+\mathrm{H}]^{+}$309.0982, found 309.0979. Anal. Calcd for $\mathrm{C}_{16} \mathrm{H}_{12} \mathrm{~N}_{4} \mathrm{O}$ (308.29): C 62.33, H 3.92, N 18.17; found: C 62.26, H 4.22, N 17.92.

1-(1,2,3,4-Tetrahydro-2,4-dioxo-3-phenyl-1-(prop-2ynyl)quinolin-3-yl)-1H-1,2,3-triazole-4-carbaldehyde $(8 b)$. Colorless crystals, $\mathrm{mp} 176-182{ }^{\circ} \mathrm{C}$ (benzene); $R_{\mathrm{f}}=0.68$ (5\% ethanol in chloroform); $R_{\mathrm{f}}=0.51$ ( $30 \%$ ethyl acetate in chloroform); ${ }^{1} \mathrm{H}$ NMR $\left(500 \mathrm{MHz}, \mathrm{CDCl}_{3}\right) \delta$ 2.33-2.37 (m, 1H), $4.52(\mathrm{dd}, 1 \mathrm{H}, J=17.8,1.4 \mathrm{~Hz}), 5.34(\mathrm{dd}$, $1 \mathrm{H}, J=17.8,1.4 \mathrm{~Hz}), 7.22-7.28(\mathrm{~m}, 1 \mathrm{H}), 7.33-7.38(\mathrm{~m}$, $1 \mathrm{H}), 7.44-7.55(\mathrm{~m}, 5 \mathrm{H}), 7.61(\mathrm{~s}, 1 \mathrm{H}), 7.64-7.71(\mathrm{~m}, 1 \mathrm{H})$, $8.05(\mathrm{~d}, 1 \mathrm{H}, J=7.7 \mathrm{~Hz}), 10.13(\mathrm{~s}, 1 \mathrm{H}) ;{ }^{13} \mathrm{C}$ NMR $(126 \mathrm{MHz}$, $\left.\mathrm{CDCl}_{3}\right) \delta 33.7,73.8,76.6,80.2,116.0,120.8,124.8,128.5$, $128.8,129.0,129.2,130.5,131.8,137.2,140.4,145.8,165.1$, 185.2, 186.9; IR $\left(\mathrm{cm}^{-1}\right): v 3237,3151,2124,1716,1682$, $1603,1469,1373,1301,1200,1170,1042,774,692$; HRMS (ESI+): $m / z$ calcd for $\mathrm{C}_{21} \mathrm{H}_{15} \mathrm{~N}_{4} \mathrm{O}_{3}{ }^{+}[\mathrm{M}+\mathrm{H}]^{+}$371.1139, found 371.1130 .

1-(1-((1-Benzyl-1H-1,2,3-triazol-4-yl)methyl)-1,2,3,4 -tetrahydro-3-methyl-2,4-dioxoquinolin-3-yl)-1H-1,2,3 -triazole-4-carbaldehyde (9a). Colorless powder, mp 63$87^{\circ} \mathrm{C} ; R_{\mathrm{f}}=0.48$ (5\% ethanol in chloroform); $R_{\mathrm{f}}=0.13$ ( $50 \%$ ethyl acetate in petroleum ether); $R_{\mathrm{f}}=0.28$ (33\% petroleum ether in ethyl acetate); ${ }^{1} \mathrm{H}$ NMR $(500 \mathrm{MHz}$, 
$\left.\mathrm{CDCl}_{3}\right), \delta 2.16\left(\mathrm{~s}, 3 \mathrm{H}, \mathrm{CH}_{3}\right), 5.28(\mathrm{~d}, 1 \mathrm{H}, J=15.7 \mathrm{~Hz}$, $\mathrm{N}-1-\mathrm{CH} \alpha), 5.37(\mathrm{~d}, 1 \mathrm{H}, J=15.7 \mathrm{~Hz}, \mathrm{~N}-1-\mathrm{CH} \beta), 5.45(\mathrm{~d}$, $\left.1 \mathrm{H}, J=14.8 \mathrm{~Hz}, \mathrm{~N}-1^{\mathrm{C}}-\mathrm{CH} \alpha\right), 5.50(\mathrm{~d}, 1 \mathrm{H}, J=14.8 \mathrm{~Hz}$, $\left.\mathrm{N}-1^{\mathrm{C}}-\mathrm{CH} \beta\right), 7.21-7.26\left(\mathrm{~m}, 2 \mathrm{H}, \mathrm{H}-2^{\mathrm{D}}, \mathrm{H}-6^{\mathrm{D}}\right), 7.27$ (ddd, $1 \mathrm{H}, J=7.6,7.5,0.9 \mathrm{~Hz}, \mathrm{H}-6), 7.31-7.38\left(\mathrm{~m}, 3 \mathrm{H}, \mathrm{H}-3^{\mathrm{D}}\right.$, $\mathrm{H}-4^{\mathrm{D}}, \mathrm{H}-5^{\mathrm{D}}$ ), $7.53\left(\mathrm{~s}, 1 \mathrm{H}, \mathrm{H}-5^{\mathrm{C}}\right.$ ), 7.75 (ddd, $1 \mathrm{H}, J=8.4,7.4$, $1.7 \mathrm{~Hz}, \mathrm{H}-7), 7.86$ (d, $1 \mathrm{H}, J=8.4 \mathrm{~Hz}, \mathrm{H}-8), 8.02$ (dd, $1 \mathrm{H}, J$ $=7.8,1.6 \mathrm{~Hz}, \mathrm{H}-5), 8.30\left(\mathrm{~s}, 1 \mathrm{H}, \mathrm{H}-5^{\mathrm{A}}\right), 10.15(\mathrm{~s}, 1 \mathrm{H}, \mathrm{CHO})$; ${ }^{13} \mathrm{C}$ NMR $\left(126 \mathrm{MHz}, \mathrm{CDCl}_{3}\right) \delta 23.8\left(\mathrm{CH}_{3}\right), 39.5(\mathrm{~N}-1-$ $\left.\mathrm{CH}_{2}\right), 54.5\left(\mathrm{~N}-1^{\mathrm{C}}-\mathrm{CH}_{2}\right), 72.6(\mathrm{C}-3), 117.0(\mathrm{C}-8), 119.0$

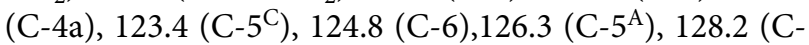
$\left.2^{\mathrm{D}}, \mathrm{C}-6^{\mathrm{D}}\right), 129.0\left(\mathrm{C}-4^{\mathrm{D}}\right), 129.3\left(\mathrm{C}-3^{\mathrm{D}}, \mathrm{C}^{\mathrm{D}} \mathrm{5}^{\mathrm{D}}\right), 129.3(\mathrm{C}-5)$, $134.3\left(\mathrm{C}-1^{\mathrm{D}}\right), 138.0(\mathrm{C}-7), 141.5(\mathrm{C}-8 \mathrm{a}), 142.7\left(\mathrm{C}-4^{\mathrm{C}}\right)$, $147.0\left(\mathrm{C}-4^{\mathrm{A}}\right), 167.7(\mathrm{C}-2), 185.0(\mathrm{CHO}), 188.9(\mathrm{C}-4) ;{ }^{15} \mathrm{~N}$ $\operatorname{NMR}\left(51 \mathrm{MHz}, \mathrm{CDCl}_{3}\right) \delta 138.4(\mathrm{~N}-1), 250.6\left(\mathrm{~N}^{-1}{ }^{\mathrm{C}}\right), 251.7$ $\left(\mathrm{N}-1^{\mathrm{A}}\right), 350.0\left(\mathrm{~N}-3^{\mathrm{C}}\right), 361.8\left(\mathrm{~N}-2^{\mathrm{A}}\right), 362.6\left(\mathrm{~N}-2^{\mathrm{C}}\right)$; IR $\left(\mathrm{cm}^{-1}\right): v 3137,2929,2852,1681,1601,1470,1385,1211$, $1186,1048,799,763,721,686,663$; HRMS (ESI+): $m / z$ calcd for $\mathrm{C}_{23} \mathrm{H}_{20} \mathrm{~N}_{7} \mathrm{O}_{3}{ }^{+}[\mathrm{M}+\mathrm{H}]^{+} 442.1622$, found 442.1620 .

1-(1,2,3,4-Tetrahydro-3-methyl-2,4-dioxo-1-((1-phenyl1H-1,2,3-triazol-4-yl)methyl)quinolin-3-yl)-1H-1,2,3triazole-4-carbaldehyde (9b). Colorless powder, mp 71$93{ }^{\circ} \mathrm{C} ; R_{\mathrm{f}}=0.44$ (33\% petroleum ether in ethyl acetate); $R_{\mathrm{f}}=0.40$ (5\% ethanol in chloroform); ${ }^{1} \mathrm{H} \mathrm{NMR}(500 \mathrm{MHz}$, $\left.\mathrm{CDCl}_{3}\right) \delta 2.22\left(\mathrm{~s}, 3 \mathrm{H}, \mathrm{CH}_{3}\right), 5.45\left(\mathrm{~s}, 2 \mathrm{H}, \mathrm{N}-1-\mathrm{CH}_{2}\right), 7.29$ (ddd, $1 \mathrm{H}, J=7.6,7.5,0.9 \mathrm{~Hz}, \mathrm{H}-6), 7.41-7.46(\mathrm{~m}, 1 \mathrm{H}$, $\left.\mathrm{H}-4^{\mathrm{D}}\right), 7.48-7.53\left(\mathrm{~m}, 2 \mathrm{H}, \mathrm{H}-3^{\mathrm{D}}, \mathrm{H}-5^{\mathrm{D}}\right), 7.68-7.72(\mathrm{~m}, 2 \mathrm{H}$, $\mathrm{H}-2^{\mathrm{D}}, \mathrm{H}-6^{\mathrm{D}}$ ), 7.78 (ddd, $\left.1 \mathrm{H}, J=8.4,7.4,1.7 \mathrm{~Hz}, \mathrm{H}-7\right), 7.90$ $(\mathrm{d}, 1 \mathrm{H}, J=8.4 \mathrm{~Hz}, \mathrm{H}-8), 8.04(\mathrm{dd}, 1 \mathrm{H}, J=7.9,1.6 \mathrm{~Hz}, \mathrm{H}-5)$, $8.05\left(\mathrm{~s}, 1 \mathrm{H}, \mathrm{H}-5^{\mathrm{C}}\right), 8.36\left(\mathrm{~s}, 1 \mathrm{H}, \mathrm{H}-5^{\mathrm{A}}\right), 10.17$ (s, $\left.1 \mathrm{H}, \mathrm{CHO}\right)$ ${ }^{13} \mathrm{C}$ NMR $\left(126 \mathrm{MHz}, \mathrm{CDCl}_{3}\right) \delta 23.8\left(\mathrm{CH}_{3}\right), \quad 39.5$ $\left(\mathrm{N}-1-\mathrm{CH}_{2}\right), 72.5$ (C-3), 117.0 (C-8), 119.0 (C-4a), 120.6 $\left(\mathrm{C}-2^{\mathrm{D}}, \mathrm{C}-6^{\mathrm{D}}\right), 121.8\left(\mathrm{C}-5^{\mathrm{C}}\right), 124.9(\mathrm{C}-6), 126.2\left(\mathrm{C}-5^{\mathrm{A}}\right)$, $129.2\left(\mathrm{C}-4^{\mathrm{D}}\right), 129.4(\mathrm{C}-5), 130.0\left(\mathrm{C}-3^{\mathrm{D}}, \mathrm{C}^{-} 5^{\mathrm{D}}\right), 136.8$ (C-1 $\left.{ }^{\mathrm{D}}\right), 138.1(\mathrm{C}-7), 141.5(\mathrm{C}-8 \mathrm{a}), 143.0\left(\mathrm{C}-4^{\mathrm{C}}\right), 147.0$ $\left(\mathrm{C}-4^{\mathrm{A}}\right), 167.8(\mathrm{C}-2), 185.0(\mathrm{CHO}), 188.8(\mathrm{C}-4) ;{ }^{15} \mathrm{~N}$ NMR $\left(51 \mathrm{MHz}, \mathrm{CDCl}_{3}\right) \delta 138.5(\mathrm{~N}-1), 251.6\left(\mathrm{~N}-1^{\mathrm{A}}\right), 256.2$ $\left(\mathrm{N}-1^{\mathrm{C}}\right), 352.0\left(\mathrm{~N}-3^{\mathrm{C}}\right), 362.2\left(\mathrm{~N}-2^{\mathrm{A}}\right)$; IR $\left(\mathrm{cm}^{-1}\right): v 3138$, 2928, 2853, 1682, 1601, 1470, 1385, 1212, 1185, 1046, 760, 690, 663; HRMS (ESI+): $m / z$ calcd for $\mathrm{C}_{22} \mathrm{H}_{18} \mathrm{~N}_{7} \mathrm{O}_{3}{ }^{+}[\mathrm{M}+$ $\mathrm{H}]^{+} 428.1466$, found 428.1461 .

1-(1,2,3,4-Tetrahydro-3-methyl-2,4-dioxo-1-((1-(pyridin-2-yl)-1H-1,2,3-triazol-4-yl)methyl)quinolin-3-yl)1H-1,2,3-triazole-4-carbaldehyde (9c). Colorless powder, $\mathrm{mp} 47-65^{\circ} \mathrm{C} ; R_{\mathrm{f}}=0.12$ (30\% ethyl acetate in chloroform); $R_{\mathrm{f}}=0.34$ (5\% ethanol in chloroform); ${ }^{1} \mathrm{H}$ NMR $(500 \mathrm{MHz}$, $\left.\mathrm{CDCl}_{3}\right) \delta 2.24\left(\mathrm{~s}, 3 \mathrm{H}, \mathrm{CH}_{3}\right), 5.35(\mathrm{~d}, 1 \mathrm{H}, J=15.9 \mathrm{~Hz}, \mathrm{~N}-1-$ $\mathrm{CH} \alpha), 5.58(\mathrm{~d}, 1 \mathrm{H}, J=15.9 \mathrm{~Hz}, \mathrm{~N}-1-\mathrm{CH} \beta), 7.24-7.31(\mathrm{~m}$, $1 \mathrm{H}, \mathrm{H}-6), 7.32-7.38\left(\mathrm{~m}, 1 \mathrm{H}, \mathrm{H}-5^{\mathrm{D}}\right), 7.76$ (ddd, $1 \mathrm{H}, J=8.4$, 7.3, $1.7 \mathrm{~Hz}, \mathrm{H}-7), 7.83$ (d, $1 \mathrm{H}, J=8.4 \mathrm{~Hz}, \mathrm{H}-8), 7.88-7.93$ $\left(\mathrm{m}, 1 \mathrm{H}, \mathrm{H}-4^{\mathrm{D}}\right), 8.04(\mathrm{dd}, 1 \mathrm{H}, J=7.8,1.6 \mathrm{~Hz}, \mathrm{H}-5), 8.11-$ $8.16\left(\mathrm{~m}, 1 \mathrm{H}, \mathrm{H}-3^{\mathrm{D}}\right), 8.35\left(\mathrm{~s}, 1 \mathrm{H}, \mathrm{H}-5^{\mathrm{A}}\right), 8.46-8.49(\mathrm{~m}, 1 \mathrm{H}$, $\left.\mathrm{H}-6^{\mathrm{D}}\right), 8.59$ (s, $\left.1 \mathrm{H}, \mathrm{H}-5^{\mathrm{C}}\right), 10.18$ (s, $\left.1 \mathrm{H}, \mathrm{CHO}\right) ;{ }^{13} \mathrm{C}$ NMR $\left(126 \mathrm{MHz}, \mathrm{CDCl}_{3}\right) \delta 24.0\left(\mathrm{CH}_{3}\right), 39.4\left(\mathrm{~N}-1-\mathrm{CH}_{2}\right), 72.9$
(C-3), $113.9\left(\mathrm{C}^{\mathrm{D}} \mathrm{3}^{\mathrm{D}}\right), 116.8$ (C-8), 119.1 (C-4a), 120.8 $\left(\mathrm{C}-5^{\mathrm{C}}\right), 124.1\left(\mathrm{C}-5^{\mathrm{D}}\right), 124.8(\mathrm{C}-6), 126.4\left(\mathrm{C}-5^{\mathrm{A}}\right), 129.4(\mathrm{C}-$ 5), $137.9(\mathrm{C}-7), 139.3\left(\mathrm{C}-4^{\mathrm{D}}\right), 141.6(\mathrm{C}-8 \mathrm{a}), 142.8\left(\mathrm{C}-4^{\mathrm{C}}\right)$, $147.1\left(\mathrm{C}-4^{\mathrm{A}}\right), 148.8\left(\mathrm{C}-6^{\mathrm{D}}\right), 148.9\left(\mathrm{C}-2^{\mathrm{D}}\right), 167.7(\mathrm{C}-2)$, 185.1 (CHO), $189.0(\mathrm{C}-4) ;{ }^{15} \mathrm{~N}$ NMR $\left(51 \mathrm{MHz}, \mathrm{CDCl}_{3}\right) \delta$ $137.8(\mathrm{~N}-1), 251.1\left(\mathrm{~N}-1^{\mathrm{A}}\right), 261.0\left(\mathrm{~N}-1^{\mathrm{C}}\right), 283.9\left(\mathrm{~N}-1^{\mathrm{D}}\right)$, $355.3\left(\mathrm{~N}-3^{\mathrm{C}}\right), 361.8\left(\mathrm{~N}-2^{\mathrm{A}}\right)$; IR $\left(\mathrm{cm}^{-1}\right): v 3138,2929,2854$, 1683, 1600, 1471, 1385, 1211, 1184, 1038, 999, 781, 760, 663; HRMS (ESI+): $m / z$ calcd for $\mathrm{C}_{21} \mathrm{H}_{17} \mathrm{~N}_{8} \mathrm{O}_{3}{ }^{+}[\mathrm{M}+\mathrm{H}]^{+}$ 429.1418 , found 429.1431 .

1-(1-((1-Benzyl-1H-1,2,3-triazol-4-yl)methyl)-1,2,3,4tetrahydro-2,4-dioxo-3-phenylquinolin-3-yl)-1H-1,2,3triazole-4-carbaldehyde (9d). Colorless powder, $\mathrm{mp} 87-$ $113{ }^{\circ} \mathrm{C} ; R_{\mathrm{f}}=0.58$ (5\% ethanol in chloroform); $R_{\mathrm{f}}=0.40$ (33\% petroleum ether in ethyl acetate); ${ }^{1} \mathrm{H}$ NMR (500 $\left.\mathrm{MHz}, \mathrm{CDCl}_{3}\right) \delta 5.20(\mathrm{~d}, 1 \mathrm{H}, J=15.6 \mathrm{~Hz}, \mathrm{~N}-1-\mathrm{CH} \alpha), 5.44$ $\left(\mathrm{d}, 1 \mathrm{H}, J=14.8 \mathrm{~Hz}, \mathrm{~N}-1^{\mathrm{C}}-\mathrm{CH} \alpha\right), 5.53(\mathrm{~d}, 1 \mathrm{H}, J=15.6 \mathrm{~Hz}$, $\mathrm{N}-1-\mathrm{CH} \beta), 5.56\left(\mathrm{~d}, 1 \mathrm{H}, J=14.8 \mathrm{~Hz}, \mathrm{~N}-1^{\mathrm{C}}-\mathrm{CH} \beta\right), 7.20$ (ddd, $1 \mathrm{H}, J=7.6,7.5,0.8 \mathrm{~Hz}, \mathrm{H}-6), 7.26-7.28(\mathrm{~m}, 4 \mathrm{H}$, $\left.\mathrm{H}-3^{\mathrm{B}}, \mathrm{H}-5^{\mathrm{B}}, \mathrm{H}-3^{\mathrm{D}}, \mathrm{H}-5^{\mathrm{D}}\right), 7.28-7.30\left(\mathrm{~m}, 2 \mathrm{H}, \mathrm{H}-2^{\mathrm{B}}, \mathrm{H}-6^{\mathrm{B}}\right)$, 7.37-7.40 (m, 3H, H-2 $\left.{ }^{\mathrm{D}}, \mathrm{H}-6^{\mathrm{D}}, \mathrm{H}-4^{\mathrm{B}}\right), 7.41-7.47(\mathrm{~m}, 1 \mathrm{H}$, $\left.\mathrm{H} 4^{\mathrm{D}}\right), 7.58\left(\mathrm{~s}, 1 \mathrm{H}, \mathrm{H}-5^{\mathrm{C}}\right), 7.58\left(\mathrm{~s}, 1 \mathrm{H}, \mathrm{H}-5^{\mathrm{A}}\right), 7.65(\mathrm{ddd}, 1 \mathrm{H}$, $J=8.4,7.4,1.7 \mathrm{~Hz}, \mathrm{H}-7), 7.78(\mathrm{~d}, 1 \mathrm{H}, J=8.4 \mathrm{~Hz}, \mathrm{H}-8), 8.00$ $(\mathrm{dd}, 1 \mathrm{H}, J=7.7,1.6 \mathrm{~Hz}, \mathrm{H}-5), 10.13(\mathrm{~s}, 1 \mathrm{H}, \mathrm{CHO}) ;{ }^{13} \mathrm{C}$ NMR $\left(126 \mathrm{MHz}, \mathrm{CDCl}_{3}\right) \delta 40.0\left(\mathrm{~N}-1-\mathrm{CH}_{2}\right), 54.5\left(\mathrm{~N}-1^{\mathrm{C}}\right.$ $\left.\mathrm{CH}_{2}\right), 80.1$ (C-3), $117.0(\mathrm{C}-8), 120.7(\mathrm{C}-4 \mathrm{a}), 123.5\left(\mathrm{C}-5^{\mathrm{C}}\right)$, $124.8(\mathrm{C}-6), 128.3\left(\mathrm{C}-3^{\mathrm{B}}, \mathrm{C}-5^{\mathrm{B}}\right), 128.4\left(\mathrm{C}-5^{\mathrm{A}}\right), 128.6\left(\mathrm{C}-3^{\mathrm{D}}\right.$, C-5 $\left.{ }^{\mathrm{D}}\right), 129.0(\mathrm{C}-5), 129.0\left(\mathrm{C}-1^{\mathrm{B}}\right), 129.1\left(\mathrm{C} 4^{\mathrm{B}}\right), 129.4\left(\mathrm{C}-2^{\mathrm{D}}\right.$, C-6 $\left.{ }^{\mathrm{D}}\right), 130.4\left(\mathrm{C}-2^{\mathrm{B}}, \mathrm{C}-6^{\mathrm{B}}\right), 131.7\left(\mathrm{C}-4^{\mathrm{D}}\right), 134.4\left(\mathrm{C}-1^{\mathrm{D}}\right)$, $137.5(\mathrm{C}-7), 141.0(\mathrm{C}-8 \mathrm{a}), 142.7\left(\mathrm{C}-4^{\mathrm{C}}\right), 145.8\left(\mathrm{C}-4^{\mathrm{A}}\right)$, 165.9 (C-2), 185.2 (CHO), $187.3(\mathrm{C}-4) ;{ }^{15} \mathrm{~N}$ NMR (51 $\left.\mathrm{MHz}, \mathrm{CDCl}_{3}\right) \delta 140.0(\mathrm{~N}-1), 250.6\left(\mathrm{~N}-1^{\mathrm{C}}\right), 253.7\left(\mathrm{~N}-1^{\mathrm{A}}\right)$, $350.8\left(\mathrm{~N}-3^{\mathrm{C}}\right), 362.5\left(\mathrm{~N}-2^{\mathrm{C}}\right)$; IR $\left(\mathrm{cm}^{-1}\right)$ : $v 3138,2850,1701$, 1680, 1601, 1469, 1376, 1044, 871, 772, 748, 724, 696; HRMS (ESI+): $m / z$ calcd for $\mathrm{C}_{28} \mathrm{H}_{22} \mathrm{~N}_{7} \mathrm{O}_{3}{ }^{+}[\mathrm{M}+\mathrm{H}]^{+}$ 504.1779, found 504.1782.

1-(1,2,3,4-Tetrahydro-2,4-dioxo-3-phenyl-1-((1-phenyl1H-1,2,3-triazol-4-yl)methyl)quinolin-3-yl)-1H-1,2,3triazole-4-carbaldehyde (9e). Colorless powder, mp 91$122{ }^{\circ} \mathrm{C} ; R_{\mathrm{f}}=0.62$ (5\% ethanol in chloroform), $R_{\mathrm{f}}=0.29$ (50\% ethyl acetate in petroleum ether); ${ }^{1} \mathrm{H}$ NMR (500 $\left.\mathrm{MHz}, \mathrm{CDCl}_{3}\right) \delta 5.41(\mathrm{~d}, 1 \mathrm{H}, J=15.7 \mathrm{~Hz}, \mathrm{~N}-1-\mathrm{CH} \alpha), 5.58$ $(\mathrm{d}, 1 \mathrm{H}, J=15.7 \mathrm{~Hz}, \mathrm{~N}-1-\mathrm{CH} \beta), 7.23$ (ddd, $1 \mathrm{H}, J=7.6,7.5$, $0.9 \mathrm{~Hz}, \mathrm{H}-6), 7.41-7.43\left(\mathrm{~m}, 2 \mathrm{H}, \mathrm{H}-2^{\mathrm{B}}, \mathrm{H}-6^{\mathrm{B}}\right), 7.43-7.45$ (m, 2H, H-3 $\left.{ }^{\mathrm{B}}, \mathrm{H}-5^{\mathrm{B}}\right), 7.45-7.48\left(\mathrm{~m}, 1 \mathrm{H}, \mathrm{H}-4^{\mathrm{D}}\right), 7.48-7.51$ $\left(\mathrm{m}, 1 \mathrm{H}, \mathrm{H}-4^{\mathrm{B}}\right), 7.51-7.55\left(\mathrm{~m}, 2 \mathrm{H}, \mathrm{H}-3^{\mathrm{D}}, \mathrm{H}-5^{\mathrm{D}}\right), 7.64(\mathrm{~s}, 1 \mathrm{H}$, H-5 ${ }^{\mathrm{A}}$ ), 7.68 (ddd, $\left.1 \mathrm{H}, J=9.5,7.4,1.7 \mathrm{~Hz}, \mathrm{H}-7\right), 7.69-7.72$ (m, 2H, H-2 $\left.2^{\mathrm{D}}, \mathrm{H}-6^{\mathrm{D}}\right), 7.79(\mathrm{~d}, 1 \mathrm{H}, J=8.4 \mathrm{~Hz}, \mathrm{H}-8), 8.04$ (dd, $1 \mathrm{H}, J=7.8,1.6 \mathrm{~Hz}, \mathrm{H}-5), 8.06\left(\mathrm{~s}, 1 \mathrm{H}, \mathrm{H}-5^{\mathrm{C}}\right.$ ), 10.15 (s, $1 \mathrm{H}, \mathrm{CHO}) ;{ }^{13} \mathrm{C}$ NMR $\left(126 \mathrm{MHz}, \mathrm{CDCl}_{3}\right) \delta 39.8(\mathrm{~N}-1-$ $\left.\mathrm{CH}_{2}\right), 80.1$ (C-3), $116.8(\mathrm{C}-8), 120.7\left(\mathrm{C}-2^{\mathrm{D}}, \mathrm{C}-6^{\mathrm{D}}\right), 120.7$ (C-4a), $121.8\left(\mathrm{C}^{\mathrm{C}} \mathrm{C}\right), 124.8(\mathrm{C}-6), 128.4\left(\mathrm{C}-5^{\mathrm{A}}\right), 128.8$ $\left(\mathrm{C}-2^{\mathrm{B}}, \mathrm{C}-6^{\mathrm{B}}\right), 129.1\left(\mathrm{C}-1^{\mathrm{B}}\right), 129.2\left(\mathrm{C}-4^{\mathrm{D}}\right), 129.3(\mathrm{C}-5)$, $130.0\left(\mathrm{C}-3^{\mathrm{D}}, \mathrm{C}-5^{\mathrm{D}}\right), 130.5\left(\mathrm{C}-3^{\mathrm{B}}, \mathrm{C}-5^{\mathrm{B}}\right), 131.8\left(\mathrm{C}-4^{\mathrm{B}}\right), 136.8$ 
$\left(\mathrm{C}-1^{\mathrm{D}}\right), 137.6(\mathrm{C}-7), 140.9(\mathrm{C}-8 \mathrm{a}), 143.0\left(\mathrm{C}-4^{\mathrm{C}}\right), 145.8$ $\left(\mathrm{C}-4^{\mathrm{A}}\right), 166.2(\mathrm{C}-2), 185.1(\mathrm{CHO}), 187.2(\mathrm{C}-4) ;{ }^{15} \mathrm{~N}$ NMR $\left(51 \mathrm{MHz}, \mathrm{CDCl}_{3}\right) \delta 139.3(\mathrm{~N}-1), 254.3\left(\mathrm{~N}-1^{\mathrm{A}}\right), 256.1(\mathrm{~N}-$ $\left.1^{\mathrm{C}}\right), 256.1\left(\mathrm{~N}-2^{\mathrm{C}}\right), 352.7\left(\mathrm{~N}-3^{\mathrm{C}}\right), 363.4\left(\mathrm{~N}-2^{\mathrm{A}}\right), \operatorname{IR}\left(\mathrm{cm}^{-1}\right): v$ $3141,2848,1701,1682,1600,1468,1376,1306,1042,872$, 772, 691, 665; HRMS (ESI+): $m / z$ calcd for $\mathrm{C}_{27} \mathrm{H}_{20} \mathrm{~N}_{7} \mathrm{O}_{3}{ }^{+}$ $[\mathrm{M}+\mathrm{H}]^{+} 490.1622$, found 490.1616 .

1-(1,2,3,4-Tetrahydro-2,4-dioxo-3-phenyl-1-((1-(pyridin-2-yl)-1H-1,2,3-triazol-4-yl)methyl)quinolin-3-yl)1H-1,2,3-triazole-4-carbaldehyde (9f). Colorless powder, $\mathrm{mp} 88-114{ }^{\circ} \mathrm{C} ; R_{\mathrm{f}}=0.44$ (5\% ethanol in chloroform), $R_{\mathrm{f}}=0.23$ (30\% ethyl acetate in chloroform); ${ }^{1} \mathrm{H}$ NMR (500 $\left.\mathrm{MHz}, \mathrm{CDCl}_{3}\right) \delta 5.31(\mathrm{~d}, 1 \mathrm{H}, J=15.8 \mathrm{~Hz}, \mathrm{~N}-1-\mathrm{CH} \alpha), 5.72$ $(\mathrm{d}, 1 \mathrm{H}, J=15.8 \mathrm{~Hz}, \mathrm{~N}-1-\mathrm{CH} \beta), 7.21$ (ddd, $1 \mathrm{H}, J=7.5,7.5$, $0.9 \mathrm{~Hz}, \mathrm{H}-6), 7.35-7.39\left(\mathrm{~m}, 1 \mathrm{H}, \mathrm{H}-5^{\mathrm{D}}\right), 7.40-7.51$ (m, 5H, $\left.\mathrm{H}-2^{\mathrm{B}}, \mathrm{H}-3^{\mathrm{B}}, \mathrm{H}-4^{\mathrm{B}}, \mathrm{H}-5^{\mathrm{B}}, \mathrm{H}-6^{\mathrm{B}}\right), 7.62-7.68\left(\mathrm{~m}, 2 \mathrm{H}, \mathrm{H}-5^{\mathrm{A}}\right.$, $\mathrm{H}-7), 7.72(\mathrm{~d}, 1 \mathrm{H}, J=8.4 \mathrm{~Hz}, \mathrm{H}-8), 7.89-7.95(\mathrm{~m}, 1 \mathrm{H}$, $\left.\mathrm{H}-4^{\mathrm{D}}\right), 8.04(\mathrm{dd}, 1 \mathrm{H}, J=7.8,1.5 \mathrm{~Hz}, \mathrm{H}-5), 8.13-8.17(\mathrm{~m}$, $\left.1 \mathrm{H}, \mathrm{H}-3^{\mathrm{D}}\right), 8.48-8.52\left(\mathrm{~m}, 1 \mathrm{H}, \mathrm{H}-6^{\mathrm{D}}\right), 8.63\left(\mathrm{~s}, 1 \mathrm{H}, \mathrm{H}-5^{\mathrm{C}}\right)$, 10.15 (s, $1 \mathrm{H}, \mathrm{CHO}) ;{ }^{13} \mathrm{C}$ NMR $\left(126 \mathrm{MHz}, \mathrm{CDCl}_{3}\right) \delta 39.9$ $\left(\mathrm{N}-1-\mathrm{CH}_{2}\right), 80.2(\mathrm{C}-3), 113.9\left(\mathrm{C}-3^{\mathrm{D}}\right), 116.7$ (C-8), 120.8 (C-4a), $120.9\left(\mathrm{C}^{-} 5^{\mathrm{C}}\right), 124.1\left(\mathrm{C}-5^{\mathrm{D}}\right), 124.8$ (C-6), $128.4(\mathrm{C}-$ $\left.5^{\mathrm{A}}\right), 128.8\left(\mathrm{C}-2^{\mathrm{B}}, \mathrm{C}-6^{\mathrm{B}}\right), 129.0\left(\mathrm{C}-1^{\mathrm{B}}\right), 129.1(\mathrm{C}-5), 130.5$ $\left(\mathrm{C}-3^{\mathrm{B}}, \mathrm{C}-5^{\mathrm{B}}\right), 131.7\left(\mathrm{C}-4^{\mathrm{B}}\right), 137.4(\mathrm{C}-7), 139.3\left(\mathrm{C}-4^{\mathrm{D}}\right)$, $141.1(\mathrm{C}-8 \mathrm{a}), 142.8\left(\mathrm{C}-4^{\mathrm{C}}\right), 145.8\left(\mathrm{C}-4^{\mathrm{A}}\right), 148.9\left(\mathrm{C}-6^{\mathrm{D}}\right)$, $149.0\left(\mathrm{C}-2^{\mathrm{D}}\right), 165.9(\mathrm{C}-2), 185.2(\mathrm{CHO}), 187.3(\mathrm{C}-4) ;{ }^{15} \mathrm{~N}$ $\operatorname{NMR}\left(51 \mathrm{MHz}, \mathrm{CDCl}_{3}\right) \delta 139.2(\mathrm{~N}-1), 254.0\left(\mathrm{~N}-1^{\mathrm{A}}\right), 260.2$ $\left(\mathrm{N}-1^{\mathrm{C}}\right), 284.4\left(\mathrm{~N}-1^{\mathrm{D}}\right), 355.5\left(\mathrm{~N}-3^{\mathrm{C}}\right), 363.1\left(\mathrm{~N}-2^{\mathrm{A}}\right)$; IR $\left(\mathrm{cm}^{-}\right.$ $\left.{ }^{1}\right)$ : v 3153, 2852, 1700, 1681, 1599, 1470, 1375, 1313, 1035, 999, 776, 750, 696; HRMS (ESI+): $m / z$ calcd for $\mathrm{C}_{26} \mathrm{H}-$ ${ }_{19} \mathrm{~N}_{8} \mathrm{O}_{3}{ }^{+}[\mathrm{M}+\mathrm{H}]^{+} 491.1575$, found 491.1578 .

General procedure for the preparation of carboxylic acids 10a,b, 11a,b and 12a-f. To a vigorously stirred icecooled solution of appropriate alcohol $(1.00 \mathrm{mmol})$ in acetone, also ice-cooled solution of chromium(VI) oxide (2.4 g, $24 \mathrm{mmol}$ unless otherwise stated) in $2 \mathrm{M} \mathrm{H}_{2} \mathrm{SO}_{4}(24 \mathrm{~mL}$ unless otherwise stated) was added during 5 minutes and stirring was continued still for the time indicated in Table 4. The original intense red color of reaction mixture changed to black. After completion of reaction (TLC), ethanol $(15 \mathrm{~mL})$ was added and the mixture was poured onto ice. After the ice melted, the solid phase was filtered off, washed with water and ethanol and dried at $50{ }^{\circ} \mathrm{C}$ affording the first part of product. The filtrate was extracted with chloroform (up to $7 \times 50 \mathrm{~mL}$, until the product was detectable in the extract by TLC), washed with water $(100 \mathrm{~mL})$, dried $\left(\mathrm{Na}_{2} \mathrm{SO}_{4}\right)$ and filtered. From the filtrate, volatile components were evaporated in vacuo, whereby the second portion of crude product was obtained. In some cases, both parts of TLC and IR pure crude product were joined together and crystalized from ethyl acetate.

1-(1,2,3,4-Tetrahydro-3-methyl-2,4-dioxoquinolin-3-yl)-1H-1,2,3-triazole-4-carboxylic acid (10a). Col- orless crystals, $\mathrm{mp} 198-201{ }^{\circ} \mathrm{C}$ (ethyl acetate); $R_{\mathrm{f}}=0.05-$ 0.37 (50\% ethanol in chloroform); ${ }^{1} \mathrm{H}$ NMR $(500 \mathrm{MHz}$, DMSO- $\left.d_{6}\right) \delta 2.14\left(\mathrm{~s}, 3 \mathrm{H}, \mathrm{CH}_{3}\right), 7.22(\mathrm{~d}, 1 \mathrm{H}, J=7.9 \mathrm{~Hz}$, $\mathrm{H}-8$ ), 7.21-7.27 (m, $1 \mathrm{H}, \mathrm{H}-6), 7.74$ (ddd, $1 \mathrm{H}, J=7.8,7.7$, $1.5 \mathrm{~Hz}, \mathrm{H}-7), 7.84(\mathrm{~d}, 1 \mathrm{H}, J=7.6 \mathrm{~Hz}, \mathrm{H}-5), 8.99(\mathrm{~s}, 1 \mathrm{H}$, H-5 $\left.{ }^{\mathrm{A}}\right), 11.45$ (s, $\left.1 \mathrm{H}, \mathrm{H}-1\right), 13.21$ (br, $\left.1 \mathrm{H}, \mathrm{COOH}\right) ;{ }^{13} \mathrm{C}$ NMR $\left(126 \mathrm{MHz}, \mathrm{DMSO}-d_{6}\right) \delta 23.4\left(\mathrm{CH}_{3}\right), 73.4(\mathrm{C}-3)$, 117.0 (C-8), 117.7 (C-4a), 123.4 (C-6), 127.6 (C-5), 130.4 $\left(\mathrm{C}-5^{\mathrm{A}}\right), 137.2(\mathrm{C}-7), 139.5\left(\mathrm{C}-4^{\mathrm{A}}\right), 141.5(\mathrm{C}-8 \mathrm{a}), 161.7$ $(\mathrm{COOH}), 168.5(\mathrm{C}-2), 190.5(\mathrm{C}-4) ;{ }^{15} \mathrm{~N}$ NMR $(51 \mathrm{MHz}$, DMSO- $\left.d_{6}\right) \delta 133.2(\mathrm{~N}-1), 250.2\left(\mathrm{~N}-1^{\mathrm{A}}\right), 357.1\left(\mathrm{~N}-3^{\mathrm{A}}\right)$, $367.5\left(\mathrm{~N}-2^{\mathrm{A}}\right)$; IR $\left(\mathrm{cm}^{-1}\right): v 3436,3141,2927,1718,1684$, $1614,1485,1392,1361,1260,1163,1020,761,665,597$; HRMS (ESI+): $m / z$ calcd for $\mathrm{C}_{13} \mathrm{H}_{11} \mathrm{~N}_{4} \mathrm{O}_{4}{ }^{+}[\mathrm{M}+\mathrm{H}]^{+}$ 287.0775, found 287.0777.

1-(1,2,3,4-Tetrahydro-2,4-dioxo-3-phenylquinolin-3-yl)1H-1,2,3-triazole-4-carboxylic acid (10b). Colorless crystals, mp 205-209 ${ }^{\circ} \mathrm{C}$ (ethyl acetate); $R_{\mathrm{f}}=0.00-0.19$ (10\% ethanol in chloroform); ${ }^{1} \mathrm{H}$ NMR $(500 \mathrm{MHz}, \mathrm{DM}-$ SO- $\left.d_{6}\right) \delta 7.07(\mathrm{~d}, 1 \mathrm{H}, J=8.0 \mathrm{~Hz}, \mathrm{H}-8), 7.16(\mathrm{ddd}, 1 \mathrm{H}, J=$ 7.7, 7.5, $1.0 \mathrm{~Hz}, \mathrm{H}-6), 7.32-7.40\left(\mathrm{~m}, 2 \mathrm{H}, \mathrm{H}-2^{\mathrm{B}}, \mathrm{H}-6^{\mathrm{B}}\right)$, 7.45-7.53 (m, 3H, H-3 $\left.{ }^{\mathrm{B}}, \mathrm{H}-4^{\mathrm{B}}, \mathrm{H}-5^{\mathrm{B}}\right), 7.62(\mathrm{ddd}, 1 \mathrm{H}, J=$ 8.2, 7.3, $1.6 \mathrm{~Hz}, \mathrm{H}-7$ ), 7.83 (dd, $1 \mathrm{H}, J=7.8,1.4 \mathrm{~Hz}, \mathrm{H}-5$ ), $8.71\left(\mathrm{~s}, 1 \mathrm{H}, \mathrm{H}-5^{\mathrm{A}}\right), 11.63(\mathrm{~s}, 1 \mathrm{H}, \mathrm{H}-1), 13.06(\mathrm{br}, 1 \mathrm{H}$, $\mathrm{COOH}) ;{ }^{13} \mathrm{C}$ NMR (126 MHz, DMSO- $\left.d_{6}\right) \delta 80.6(\mathrm{C}-3)$, 116.7 (C-8), 119.6 (C-4a), 123.4 (C-6), 127.4 (C-5), 128.9 $\left(\mathrm{C}-2^{\mathrm{B}}, \mathrm{C}-6^{\mathrm{B}}\right), 129.6\left(\mathrm{C}-3^{\mathrm{B}}, \mathrm{C}-5^{\mathrm{B}}\right), 129.8\left(\mathrm{C}-1^{\mathrm{B}}\right), 130.6(\mathrm{C}-$ $\left.4^{\mathrm{B}}\right), 131.1\left(\mathrm{C}-5^{\mathrm{A}}\right), 136.7(\mathrm{C}-7), 139.2\left(\mathrm{C}-4^{\mathrm{A}}\right), 140.4(\mathrm{C}-8 \mathrm{a})$, $161.7(\mathrm{COOH}), 166.8(\mathrm{C}-2), 188.6(\mathrm{C}-4)$; IR $\left(\mathrm{cm}^{-1}\right)$ : v 3364, 3157, 1740, 1724, 1679, 1613, 1594, 1485, 1201, 1183, 1039, 855, 778, 754; HRMS (ESI+): $m / z$ calcd for $\mathrm{C}_{18} \mathrm{H}_{13} \mathrm{~N}_{4} \mathrm{O}_{4}{ }^{+}$ $[\mathrm{M}+\mathrm{H}]^{+} 349.0931$, found 349.0927 .

1-(1,2,3,4-Tetrahydro-3-methyl-2,4-dioxo-1-(prop-2ynyl)quinolin-3-yl)-1H-1,2,3-triazole-4-carboxylic acid (11a). Colorless crystals, mp $187-190{ }^{\circ} \mathrm{C}$ (ethyl acetate); $R_{\mathrm{f}}=0.15$ (50\% ethanol in chloroform); ${ }^{1} \mathrm{H}$ NMR $(500 \mathrm{MHz}$, DMSO- $\left.d_{6}\right) \delta 2.14(\mathrm{~s}, 3 \mathrm{H}), 3.38(\mathrm{dd}, 1 \mathrm{H}, J=2.4,2.3 \mathrm{~Hz}), 4.84$ (dd, $1 \mathrm{H}, J=18.1,2.3 \mathrm{~Hz}), 4.97(\mathrm{dd}, 1 \mathrm{H}, J=18.1,2.4 \mathrm{~Hz})$, $7.38(\mathrm{dd}, 1 \mathrm{H}, J=7.7,7.3 \mathrm{~Hz}), 7.59(\mathrm{~d}, 1 \mathrm{H}, J=8.5 \mathrm{~Hz}), 7.91$ (ddd, $1 \mathrm{H}, J=8.5,7.3,1.4 \mathrm{~Hz}), 7.97(\mathrm{dd}, 1 \mathrm{H}, J=7.7,1.4 \mathrm{~Hz})$, 8.99 (s, $1 \mathrm{H}), 13.23(\mathrm{br}, 1 \mathrm{H}) ;{ }^{13} \mathrm{C}$ NMR $(126 \mathrm{MHz}$, DMSO- $\left.d_{6}\right) \delta 23.5,32.7,73.7,75.4,78.2,116.7,119.2,124.2$, $128.1,130.6,137.1,139.6,140.7,161.7,167.7,189.5$; IR $\left(\mathrm{cm}^{-1}\right): v 3259,3137,2127,1742,1693,1650,1603,1472$, 1393, 1304, 1214, 1187, 1045, 781, 753; HRMS (ESI+): $\mathrm{m} / z$ calcd for $\mathrm{C}_{16} \mathrm{H}_{13} \mathrm{~N}_{4} \mathrm{O}_{4}{ }^{+}[\mathrm{M}+\mathrm{H}]^{+}$325.0931, found 325.0930.

1-(1,2,3,4-Tetrahydro-2,4-dioxo-3-phenyl-1-(prop-2ynyl)quinolin-3-yl)-1H-1,2,3-triazole-4-carboxylic acid (11b). Colorless crystals, mp $154-161{ }^{\circ} \mathrm{C}$ (ethyl acetate); $R_{\mathrm{f}}=0.23$ (50\% ethanol in chloroform); $R_{\mathrm{f}}=0.00-0.15$ (10\% ethanol in chloroform); ${ }^{1} \mathrm{H}$ NMR $(500 \mathrm{MHz}$, DMSO- $\left.d_{6}\right) \delta 3.40-3.44(\mathrm{~m}, 1 \mathrm{H}), 4.80(\mathrm{dd}, 1 \mathrm{H}, J=16.8$, $3.3 \mathrm{~Hz}), 5.16(\mathrm{dd}, 1 \mathrm{H}, J=16.8,3.3 \mathrm{~Hz}), 7.23-7.32(\mathrm{~m}, 3 \mathrm{H})$, 
7.39-7.53 (m, 4H), 7.75 (ddd, $1 \mathrm{H}, J=8.4,7.4,1.7 \mathrm{~Hz}), 7.92$ (dd, $1 \mathrm{H}, J=7.7,1.5 \mathrm{~Hz}), 8.79(\mathrm{~s}, 1 \mathrm{H}), 13.23(\mathrm{br}, 1 \mathrm{H}) ;{ }^{13} \mathrm{C}$ NMR $\left(126 \mathrm{MHz}, \mathrm{DMSO}-d_{6}\right) \delta 33.2,75.5,77.8,80.5,116.3$, $121.0,124.2,127.8,128.7,129.5,129.7,130.7,131.3,136.7$, 139.2, 139.9, 161.7, 165.7, 187.5; IR $\left(\mathrm{cm}^{-1}\right): v$ 3494, 3205, 2118, 1720, 1683, 1603, 1469, 1374, 1306, 1218, 1040, 871, 764, 696; HRMS (ESI+): $m / z$ calcd for $\mathrm{C}_{21} \mathrm{H}_{15} \mathrm{~N}_{4} \mathrm{O}_{4}{ }^{+}[\mathrm{M}+$ $\mathrm{H}]^{+}$387.1088, found 387.1084 .

1-(1-((1-Benzyl-1H-1,2,3-triazol-4-yl)methyl)-1,2,3,4 -tetrahydro-3-methyl-2,4-dioxoquinolin-3-yl)-1H -1,2,3-triazole-4-carboxylic acid (12a). Colorless solid, $\mathrm{mp} 129-148{ }^{\circ} \mathrm{C} ; R_{\mathrm{f}}=0.00-0.35$ (10\% ethanol in chloroform); ${ }^{1} \mathrm{H}$ NMR (500 MHz, DMSO- $d_{6}$ ), $\delta 2.15$ (s, $3 \mathrm{H}$, $\left.\mathrm{CH}_{3}\right), 5.18(\mathrm{~d}, 1 \mathrm{H}, J=16.2 \mathrm{~Hz}, \mathrm{~N}-1-\mathrm{CH} \alpha), 5.48(\mathrm{~d}, 1 \mathrm{H}, J=$ $16.2 \mathrm{~Hz}, \mathrm{~N}-1-\mathrm{CH} \beta), 5.57\left(\mathrm{~s}, 2 \mathrm{H}, \mathrm{N}-1^{\mathrm{C}}-\mathrm{CH}_{2}\right), 7.24-7.28$ (m, 2H, H-2 ${ }^{\mathrm{D}}, \mathrm{H}-6^{\mathrm{D}}$ ), 7.28-7.38 (m, 4H, H-6, H-3 ${ }^{\mathrm{D}}, \mathrm{H}-4^{\mathrm{D}}$, H-5 $\left.{ }^{\mathrm{D}}\right), 7.64(\mathrm{~d}, 1 \mathrm{H}, J=8.5 \mathrm{~Hz}, \mathrm{H}-8), 7.81$ (ddd, $1 \mathrm{H}, J=8.7$, 7.1, $1.7 \mathrm{~Hz}, \mathrm{H}-7$ ), 7.93 (dd, $1 \mathrm{H}, J=7.6,1.2 \mathrm{~Hz}, \mathrm{H}-5$ ), 8.16 (s, $\left.1 \mathrm{H}, \mathrm{H}-5^{\mathrm{C}}\right), 8.96\left(\mathrm{~s}, 1 \mathrm{H}, \mathrm{H}-5^{\mathrm{A}}\right), 12.98$ (br, $\left.1 \mathrm{H}, \mathrm{COOH}\right)$; ${ }^{13} \mathrm{C}$ NMR $\left(126 \mathrm{MHz}, \mathrm{DMSO}-d_{6}\right) \delta 23.6\left(\mathrm{CH}_{3}\right), 38.9(\mathrm{~N}-1-$ $\left.\mathrm{CH}_{2}\right), 52.9\left(\mathrm{~N}-1^{\mathrm{C}}-\mathrm{CH}_{2}\right), 74.0(\mathrm{C}-3), 116.7(\mathrm{C}-8), 119.3$ (C-4a), $123.9\left({\mathrm{C}-5^{\mathrm{C}}}^{\mathrm{C}}\right), 123.9(\mathrm{C}-6), 127.9\left(\mathrm{C}-2^{\mathrm{D}}, \mathrm{C}-6^{\mathrm{D}}\right)$, $128.0\left(\mathrm{C}-4^{\mathrm{D}}\right), \quad 128.2(\mathrm{C}-5), 128.8\left(\mathrm{C}-3^{\mathrm{D}}, \mathrm{C}^{\mathrm{D}} \mathrm{5}^{\mathrm{D}}\right), 130.6$ $\left(\mathrm{C}-5^{\mathrm{A}}\right), 136.0\left(\mathrm{C}-1^{\mathrm{D}}\right), 137.1(\mathrm{C}-7), 139.6\left(\mathrm{C}-4^{\mathrm{A}}\right), 141.4$ (C-8a), $142.3\left(\mathrm{C}-4^{\mathrm{C}}\right), 161.7(\mathrm{COOH}), 168.2(\mathrm{C}-2), 189.8$ (C-4); ${ }^{15} \mathrm{~N}$ NMR $\left(51 \mathrm{MHz}, \mathrm{DMSO}-d_{6}\right) \delta 136.8(\mathrm{~N}-1), 249.3$ $\left(\mathrm{N}-1^{\mathrm{A}}\right), 251.2\left(\mathrm{~N}-1^{\mathrm{C}}\right), 351.1\left(\mathrm{~N}-3^{\mathrm{C}}\right), 357.4\left(\mathrm{~N}-3^{\mathrm{A}}\right), 362.4$ $\left(\mathrm{N}-2^{\mathrm{C}}\right), 367.7\left(\mathrm{~N}-2^{\mathrm{A}}\right)$; IR $\left(\mathrm{cm}^{-1}\right): v$ 3468, 3140, 2945, 1716, 1679, 1602, 1470, 1385, 1278, 1222, 1045, 784, 764, 721; HRMS (ESI+): $m / z$ calcd for $\mathrm{C}_{23} \mathrm{H}_{20} \mathrm{~N}_{7} \mathrm{O}_{4}{ }^{+}[\mathrm{M}+\mathrm{H}]^{+}$ 458.1571 , found 458.1579 .

1-(1,2,3,4-Tetrahydro-3-methyl-2,4-dioxo-1-((1-phenyl1H-1,2,3-triazol-4-yl)methyl)quinolin-3-yl)-1H-1,2,3triazole-4-carboxylic acid (12b). Colorless solid, mp 143$168{ }^{\circ} \mathrm{C} ; R_{\mathrm{f}}=0.00-0.35$ (10\% ethanol in chloroform); $R_{\mathrm{f}}=0.18$ (50\% ethanol in chloroform); ${ }^{1} \mathrm{H}$ NMR (500 $\left.\mathrm{MHz}, \mathrm{DMSO}-d_{6}\right) \delta 2.22\left(\mathrm{~s}, 3 \mathrm{H}, \mathrm{CH}_{3}\right), 5.27(\mathrm{~d}, 1 \mathrm{H}, J=16.3$ $\mathrm{Hz}, \mathrm{N}-1-\mathrm{CH} \alpha), 5.62(\mathrm{~d}, 1 \mathrm{H}, J=16.3 \mathrm{~Hz}, \mathrm{~N}-1-\mathrm{CH} \beta), 7.34$ (dd, $1 \mathrm{H}, J=7.4,7.4 \mathrm{~Hz}, \mathrm{H}-6), 7.45-7.52\left(\mathrm{~m}, 1 \mathrm{H}, \mathrm{H}-4^{\mathrm{D}}\right)$, 7.55-7.62 (m, 2H, H-3 $\left.{ }^{\mathrm{D}}, \mathrm{H}-5^{\mathrm{D}}\right), 7.69(\mathrm{~d}, 1 \mathrm{H}, J=8.4 \mathrm{~Hz}$, H-8), 7.81-7.91 (m, 3H, H-7, H-2 $\left.{ }^{\mathrm{D}}, \mathrm{H}-6^{\mathrm{D}}\right), 7.96(\mathrm{~d}, 1 \mathrm{H}, J=$ $7.3 \mathrm{~Hz}, \mathrm{H}-5), 8.74\left(\mathrm{~s}, 1 \mathrm{H}, \mathrm{H}-5^{\mathrm{C}}\right), 8.97\left(\mathrm{~s}, 1 \mathrm{H}, \mathrm{H}-5^{\mathrm{A}}\right), 13.02$ (br, $1 \mathrm{H}, \mathrm{COOH}) ;{ }^{13} \mathrm{C}$ NMR $\left(126 \mathrm{MHz}, \mathrm{DMSO}-d_{6}\right) \delta 23.6$ $\left(\mathrm{CH}_{3}\right), 38.8\left(\mathrm{~N}-1-\mathrm{CH}_{2}\right), 74.1(\mathrm{C}-3), 116.8(\mathrm{C}-8), 119.4$ (C-4a), $120.2\left(\mathrm{C}-2^{\mathrm{D}}, \mathrm{C}-6^{\mathrm{D}}\right), 121.8\left(\mathrm{C}-5^{\mathrm{C}}\right), 124.0(\mathrm{C}-6)$, $128.0(\mathrm{C}-5), 128.9\left(\mathrm{C}-4^{\mathrm{D}}\right), 130.0\left(\mathrm{C}-3^{\mathrm{D}}, \mathrm{C}-5^{\mathrm{D}}\right), 130.6(\mathrm{C}-$ $\left.5^{\mathrm{A}}\right), 136.5\left(\mathrm{C}-1^{\mathrm{D}}\right), 137.2(\mathrm{C}-7), 139.7\left(\mathrm{C}-4^{\mathrm{A}}\right), 141.5(\mathrm{C}-8 \mathrm{a})$, $143.3\left(\mathrm{C}-4^{\mathrm{C}}\right), 161.7(\mathrm{COOH}), 168.3(\mathrm{C}-2), 189.9(\mathrm{C}-4) ;{ }^{15} \mathrm{~N}$ NMR $\left(51 \mathrm{MHz}, \mathrm{DMSO}-d_{6}\right) \delta 135.9(\mathrm{~N}-1), 249.4\left(\mathrm{~N}-1^{\mathrm{A}}\right)$, $255.8\left(\mathrm{~N}-1^{\mathrm{C}}\right), 353.6\left(\mathrm{~N}-3^{\mathrm{C}}\right), 356.9\left(\mathrm{~N}-3^{\mathrm{A}}\right), 358.2\left(\mathrm{~N}-2^{\mathrm{C}}\right)$, $367.2\left(\mathrm{~N}-2^{\mathrm{A}}\right)$; IR $\left(\mathrm{cm}^{-1}\right): v 3142,3085,2925,1717,1679$, 1601, 1470, 1386, 1278, 1226, 1193, 1045, 760, 690, 663; HRMS (ESI+): $m / z$ calcd for $\mathrm{C}_{22} \mathrm{H}_{18} \mathrm{~N}_{7} \mathrm{O}_{4}{ }^{+}[\mathrm{M}+\mathrm{H}]^{+}$ 444.1415, found 444.1413.
1-(1,2,3,4-Tetrahydro-3-methyl-2,4-dioxo-1-((1-(pyridin-2-yl)-1H-1,2,3-triazol-4-yl)methyl)quinolin-3-yl)1H-1,2,3-triazole-4-carboxylic acid (12c). Colorless solid, $\mathrm{mp} 152-161{ }^{\circ} \mathrm{C} ; R_{\mathrm{f}}=0.03$ ( $10 \%$ ethanol in chloroform); $R_{\mathrm{f}}=0.14$ (50\% ethanol in chloroform); ${ }^{1} \mathrm{H}$ NMR (500 $\left.\mathrm{MHz}, \mathrm{DMSO}-d_{6}\right) \delta 2.22(\mathrm{~s}, 3 \mathrm{H}), 5.40(\mathrm{~d}, 1 \mathrm{H}, J=16.5 \mathrm{~Hz})$, $5.55(\mathrm{~d}, 1 \mathrm{H}, J=16.5 \mathrm{~Hz}), 7.33$ (ddd, $1 \mathrm{H}, J=7.6,7.4,0.8$ $\mathrm{Hz}), 7.51-7.57(\mathrm{~m}, 1 \mathrm{H}), 7.61(\mathrm{~d}, 1 \mathrm{H}, J=8.5 \mathrm{~Hz}), 7.81(\mathrm{ddd}$, $1 \mathrm{H}, J=8.4,7.4,1.7 \mathrm{~Hz}), 7.96(\mathrm{dd}, 1 \mathrm{H}, J=7.7,1.6 \mathrm{~Hz})$, 8.09-8.13 (m, 2H), 8.56-8.60 (m, 1H), $8.83(\mathrm{~s}, 1 \mathrm{H}), 8.99(\mathrm{~s}$, $1 \mathrm{H}), 13.19$ (br, $1 \mathrm{H}) ;{ }^{13} \mathrm{C}$ NMR (126 MHz, DMSO- $\left.d_{6}\right) \delta$ $23.6\left(\mathrm{CH}_{3}\right), 38.8\left(\mathrm{~N}-1-\mathrm{CH}_{2}\right), 74.2(\mathrm{C}-3), 113.7\left(\mathrm{C}-3^{\mathrm{D}}\right)$ 116.6 (C-8), 119.4 (C-4a), $120.7\left(\mathrm{C}-5^{\mathrm{C}}\right), 123.9$ (C-6), 124.5 $\left(\mathrm{C}-5^{\mathrm{D}}\right), 128.0(\mathrm{C}-5), 130.6\left(\mathrm{C}-5^{\mathrm{A}}\right), 137.1(\mathrm{C}-7), 139.7$ $\left(\mathrm{C}-4^{\mathrm{A}}\right), 140.2\left(\mathrm{C}-4^{\mathrm{D}}\right), 141.2(\mathrm{C}-8 \mathrm{a}), 143.2\left(\mathrm{C}-4^{\mathrm{C}}\right), 148.4$ $\left(\mathrm{C}-2^{\mathrm{D}}\right), 149.0\left(\mathrm{C}-6^{\mathrm{D}}\right), 161.7(\mathrm{COOH}), 168.5(\mathrm{C}-2), 189.8$ $(\mathrm{C}-4) ;{ }^{15} \mathrm{~N}$ NMR $\left(51 \mathrm{MHz}, \mathrm{DMSO}-d_{6}\right) 1 \delta 249.2\left(\mathrm{~N}-1^{\mathrm{A}}\right)$, $260.3\left(\mathrm{~N}-1^{\mathrm{C}}\right), 284.1\left(\mathrm{~N}-1^{\mathrm{D}}\right)$; IR $\left(\mathrm{cm}^{-1}\right): v 3147,2926,1717$, 1680, 1600, 1471, 1385, 1278, 1223, 1038, 1000, 781, 755, 663; HRMS (ESI+): $m / z$ calcd for $\mathrm{C}_{21} \mathrm{H}_{17} \mathrm{~N}_{8} \mathrm{O}_{4}{ }^{+}[\mathrm{M}+\mathrm{H}]^{+}$ 445.1367, found 445.1372.

1-(1-((1-Benzyl-1H-1,2,3-triazol-4-yl)methyl)-1,2,3,4tetrahydro-2,4-dioxo-3-phenylquinolin-3-yl)-1H-1,2,3triazole-4-carboxylic acid (12d). Colorless solid, mp 139$162{ }^{\circ} \mathrm{C} ; R_{\mathrm{f}}=0.12-0.46$ (10\% ethanol in chloroform); $R_{\mathrm{f}}=0.00-0.18$ (3\% ethanol in chloroform); ${ }^{1} \mathrm{H}$ NMR (500 MHz, DMSO- $\left.d_{6}\right) \delta 5.11(\mathrm{~d}, 1 \mathrm{H}, J=15.9 \mathrm{~Hz}, \mathrm{~N}-1-\mathrm{CH} \alpha)$, $5.61(\mathrm{~d}, 1 \mathrm{H}, J=15.9 \mathrm{~Hz}, \mathrm{~N}-1-\mathrm{CH} \beta), 5.61(\mathrm{~d}, 2 \mathrm{H}, J=14.8$ $\left.\mathrm{Hz}, \mathrm{N}-1^{\mathrm{C}}-\mathrm{CH}_{2}\right), 7.13-7.20\left(\mathrm{~m}, 2 \mathrm{H}, \mathrm{H}-2^{\mathrm{B}}, \mathrm{H}-6^{\mathrm{B}}\right), 7.21-7.29$ (m, 3H, H-6, H-3 $\left.{ }^{\mathrm{B}}, \mathrm{H}-5^{\mathrm{B}}\right), 7.29-7.44\left(\mathrm{~m}, 6 \mathrm{H}, \mathrm{H}-2^{\mathrm{D}}, \mathrm{H}-6^{\mathrm{D}}\right.$, $\left.\mathrm{H}-4^{\mathrm{D}}, \mathrm{H}-3^{\mathrm{D}}, \mathrm{H}-5^{\mathrm{D}}, \mathrm{H}-4^{\mathrm{B}}\right), 7.66(\mathrm{~d}, 1 \mathrm{H}, J=8.2 \mathrm{~Hz}, \mathrm{H}-8)$, $7.71(\mathrm{dd}, 1 \mathrm{H}, J=8.0,7.9 \mathrm{~Hz}, \mathrm{H}-7), 7.90(\mathrm{~d}, 1 \mathrm{H}, J=7.4 \mathrm{~Hz}$, $\mathrm{H}-5), 8.24\left(\mathrm{~s}, 1 \mathrm{H}, \mathrm{H}-5^{\mathrm{C}}\right), 8.76\left(\mathrm{~s}, 1 \mathrm{H}, \mathrm{H}-5^{\mathrm{A}}\right), 13.25$ (br, $1 \mathrm{H}$, $\mathrm{COOH}) ;{ }^{13} \mathrm{C}$ NMR $\left(126 \mathrm{MHz}, \mathrm{DMSO}-d_{6}\right) \delta 39.8(\mathrm{~N}-1-$ $\left.\mathrm{CH}_{2}\right), 52.8\left(\mathrm{~N}-1^{\mathrm{C}}-\mathrm{CH}_{2}\right), 80.5(\mathrm{C}-3), 116.6(\mathrm{C}-8), 121.1$

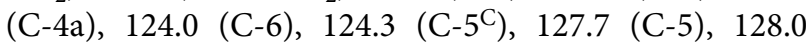
$\left(\mathrm{C}-2^{\mathrm{D}}, \mathrm{C}-6^{\mathrm{D}}\right), 128.2\left(\mathrm{C}-4^{\mathrm{D}}\right), 128.7\left(\mathrm{C}-2^{\mathrm{B}}, \mathrm{C}-6^{\mathrm{B}}\right), 128.8$ $\left(\mathrm{C}-3^{\mathrm{D}}, \mathrm{C}-5^{\mathrm{D}}\right), 129.3\left(\mathrm{C}-3^{\mathrm{B}}, \mathrm{C}-5^{\mathrm{B}}\right), 129.6\left(\mathrm{C}-1^{\mathrm{B}}\right), 130.5$ $\left(\mathrm{C}-4^{\mathrm{B}}\right), 131.2\left(\mathrm{C}-5^{\mathrm{A}}\right), 136.0\left(\mathrm{C}-1^{\mathrm{D}}\right), 136.6(\mathrm{C}-7), 139.3$ $\left(\mathrm{C}-4^{\mathrm{A}}\right), 140.8(\mathrm{C}-8 \mathrm{a}), 141.9\left(\mathrm{C}-4^{\mathrm{C}}\right), 161.7(\mathrm{COOH}), 166.1$ (C-2), $187.9(\mathrm{C}-4) ;{ }^{15} \mathrm{~N}$ NMR (51 MHz, DMSO- $\left.d_{6}\right) \delta 249.8$ $\left(\mathrm{N}-1^{\mathrm{A}}\right), 250.9\left(\mathrm{~N}-1^{\mathrm{C}}\right), 351.6\left(\mathrm{~N}-3^{\mathrm{C}}\right), 356.4\left(\mathrm{~N}-3^{\mathrm{A}}\right), 362.7$ $\left(\mathrm{N}-2^{\mathrm{C}}\right)$; IR $\left(\mathrm{cm}^{-1}\right): v 3467,3141,1717,1680,1601,1469$, 1376, 1224, 1038, 872, 760, 724, 696, 665, 610; HRMS (ESI+): $m / z$ calcd for $\mathrm{C}_{28} \mathrm{H}_{22} \mathrm{~N}_{7} \mathrm{O}_{4}{ }^{+}[\mathrm{M}+\mathrm{H}]^{+} 520.1728$, found 520.1730 .

1-(1,2,3,4-Tetrahydro-2,4-dioxo-3-phenyl-1-((1-phenyl1H-1,2,3-triazol-4-yl)methyl)quinolin-3-yl)-1H-1,2,3triazole-4-carboxylic acid (12e). Colorless solid, mp 153$169^{\circ} \mathrm{C} ; R_{\mathrm{f}}=0.00-0.22$ (10\% ethanol in chloroform); ${ }^{1} \mathrm{H}$ NMR $\left(500 \mathrm{MHz}\right.$, DMSO- $\left.d_{6}\right) \delta 5.28(\mathrm{~d}, 1 \mathrm{H}, J=16.0 \mathrm{~Hz}$, $\mathrm{N}-1-\mathrm{CH} \alpha), 5.70(\mathrm{~d}, 1 \mathrm{H}, J=16.0 \mathrm{~Hz}, \mathrm{~N}-1-\mathrm{CH} \beta), 7.26(\mathrm{dd}$, $1 \mathrm{H}, J=7.4,7.3 \mathrm{~Hz}, \mathrm{H}-6), 7.28-7.34\left(\mathrm{~m}, 2 \mathrm{H}, \mathrm{H}-2^{\mathrm{B}}, \mathrm{H}-6^{\mathrm{B}}\right)$, 7.35-7.41 (m, 2H, H-3 $\left.3^{\mathrm{B}}, \mathrm{H}-5^{\mathrm{B}}\right), 7.41-7.46\left(\mathrm{~m}, 1 \mathrm{H}, \mathrm{H}-4^{\mathrm{B}}\right)$, 
7.48-7.54 (m, 1H, H-4 $\left.4^{\mathrm{D}}\right), 7.57-7.65\left(\mathrm{~m}, 2 \mathrm{H}, \mathrm{H}-3^{\mathrm{D}}, \mathrm{H}-5^{\mathrm{D}}\right)$, $7.68(\mathrm{~d}, 1 \mathrm{H}, J=8.3 \mathrm{~Hz}, \mathrm{H}-8), 7.73(\mathrm{dd}, 1 \mathrm{H}, J=7.6,7.4 \mathrm{~Hz}$, H-7), 7.85-7.91 (m, 2H, H2 $\left.{ }^{\mathrm{D}}, \mathrm{H}-6^{\mathrm{D}}\right), 7.93(\mathrm{~d}, 1 \mathrm{H}, J=7.4$ $\mathrm{Hz}, \mathrm{H}-5), 8.79$ (s, $\left.1 \mathrm{H}, \mathrm{H}-5^{\mathrm{A}}\right), 8.81\left(\mathrm{~s}, 1 \mathrm{H}, \mathrm{H}-5^{\mathrm{C}}\right), 13.12$ (br, $1 \mathrm{H}, \mathrm{COOH}) ;{ }^{13} \mathrm{C}$ NMR $\left(126 \mathrm{MHz}, \mathrm{DMSO}-d_{6}\right) \delta 39.2$ $\left(\mathrm{N}-1-\mathrm{CH}_{2}\right), 80.7$ (C-3), $116.7(\mathrm{C}-8), 120.2\left(\mathrm{C}-2^{\mathrm{D}}, \mathrm{C}-6^{\mathrm{D}}\right)$, 121.1 (C-4a), $122.4\left(\mathrm{C}-5^{\mathrm{C}}\right), 124.1(\mathrm{C}-6), 127.8(\mathrm{C}-5), 128.9$ $\left(\mathrm{C}-4^{\mathrm{D}}\right), 129.0\left(\mathrm{C}-2^{\mathrm{B}}, \mathrm{C}-6^{\mathrm{B}}\right), 129.4\left(\mathrm{C}-3^{\mathrm{B}}, \mathrm{C}-5^{\mathrm{B}}\right), 129.7$ $\left(\mathrm{C}-1^{\mathrm{B}}\right), 130.0\left(\mathrm{C}-3^{\mathrm{D}}, \mathrm{C}-5^{\mathrm{D}}\right), 130.7\left(\mathrm{C}-4^{\mathrm{B}}\right), 131.3\left(\mathrm{C}-5^{\mathrm{A}}\right)$, $136.5\left(\mathrm{C}-1^{\mathrm{D}}\right), 136.8(\mathrm{C}-7), 139.3\left(\mathrm{C}-4^{\mathrm{A}}\right), 140.7(\mathrm{C}-8 \mathrm{a})$, $142.9\left(\mathrm{C}-4^{\mathrm{C}}\right), 161.8(\mathrm{COOH}), 166.3(\mathrm{C}-2), 188.0(\mathrm{C}-4) ;{ }^{15} \mathrm{~N}$ NMR $\left(51 \mathrm{MHz}\right.$, DMSO- $\left.d_{6}\right) \delta 139.5(\mathrm{~N}-1), 249.8\left(\mathrm{~N}-1^{\mathrm{A}}\right)$, $255.6\left(\mathrm{~N}-1^{\mathrm{C}}\right), 354.2\left(\mathrm{~N}-3^{\mathrm{C}}\right), 357.5\left(\mathrm{~N}-3^{\mathrm{A}}\right), 372.6\left(\mathrm{~N}-2^{\mathrm{A}}\right)$; IR $\left(\mathrm{cm}^{-1}\right): v 3525,3145,3067,1717,1681,1600,1469,1377$, 1234, 1041, 872, 759, 692, 665; HRMS (ESI+): $m / z$ calcd for $\mathrm{C}_{27} \mathrm{H}_{20} \mathrm{~N}_{7} \mathrm{O}_{4}{ }^{+}[\mathrm{M}+\mathrm{H}]^{+}$506.1571, found 506.1567.

1-(1,2,3,4-Tetrahydro-2,4-dioxo-3-phenyl-1-((1-(pyridin-2-yl)-1H-1,2,3-triazol-4-yl)methyl)quinolin-3-yl)1H-1,2,3-triazole-4-carboxylic acid (12f). Colorless solid, $\mathrm{mp} 116-172{ }^{\circ} \mathrm{C} ; R_{\mathrm{f}}=0.06$ (10\% ethanol in chloroform), $R_{\mathrm{f}}=0.00$ (5\% ethanol in chloroform); ${ }^{1} \mathrm{H} \mathrm{NMR} \mathrm{(500} \mathrm{MHz,}$ DMSO- $\left.d_{6}\right) \delta 5.43(\mathrm{~d}, 1 \mathrm{H}, J=16.2 \mathrm{~Hz}, \mathrm{~N}-1-\mathrm{CH} \alpha), 5.64(\mathrm{~d}$, $1 \mathrm{H}, J=16.2 \mathrm{~Hz}, \mathrm{~N}-1-\mathrm{CH} \beta), 7.25(\mathrm{dd}, 1 \mathrm{H}, J=7.4,7.3 \mathrm{~Hz}$, H-6), 7.28-7.36 (m, 2H, H-2 $\left.{ }^{\mathrm{B}}, \mathrm{H}-6^{\mathrm{B}}\right), 7.38-7.48(\mathrm{~m}, 3 \mathrm{H}$, $\left.\mathrm{H}-3^{\mathrm{B}}, \mathrm{H}-4^{\mathrm{B}}, \mathrm{H}-5^{\mathrm{B}}\right), 7.50-7.59\left(\mathrm{~m}, 2 \mathrm{H}, \mathrm{H}-8, \mathrm{H}-5^{\mathrm{D}}\right), 7.69$ (dd, $1 \mathrm{H}, J=7.6,7.5 \mathrm{~Hz}, \mathrm{H}-7), 7.95(\mathrm{~d}, 1 \mathrm{H}, J=7.4 \mathrm{~Hz}, \mathrm{H}-5)$, 8.07-8.19 (m, $\left.2 \mathrm{H}, \mathrm{H}-3^{\mathrm{D}}, \mathrm{H}-4^{\mathrm{D}}\right), 8.56-8.64\left(\mathrm{~m}, 1 \mathrm{H}, \mathrm{H}-6^{\mathrm{D}}\right)$, 8.79-8.91 (m, $\left.2 \mathrm{H}, \mathrm{H}-5^{\mathrm{C}}, \mathrm{H}-5^{\mathrm{A}}\right), 13.23(\mathrm{br}, 1 \mathrm{H}, \mathrm{COOH}) ;{ }^{13} \mathrm{C}$ NMR $\left(126 \mathrm{MHz}, \mathrm{DMSO}-d_{6}\right) \delta 39.7\left(\mathrm{~N}-1-\mathrm{CH}_{2}\right), 80.8$ (C-3), $113.7\left(\mathrm{C}-3^{\mathrm{D}}\right), 116.5(\mathrm{C}-8), 120.9\left(\mathrm{C}-5^{\mathrm{C}}\right), 121.2$ (C-4a), 124.0 (C-6), $124.5\left(\mathrm{C}-5^{\mathrm{D}}\right), 127.9$ (C-5), 128.9 $\left(\mathrm{C}-2^{\mathrm{B}}, \mathrm{C}-6^{\mathrm{B}}\right), 129.5\left(\mathrm{C}-3^{\mathrm{B}}, \mathrm{C}-5^{\mathrm{B}}\right), 129.8\left(\mathrm{C}-1^{\mathrm{B}}\right), 130.7$ $\left(\mathrm{C}-4^{\mathrm{B}}\right), 131.3\left(\mathrm{C}-5^{\mathrm{A}}\right), 136.7(\mathrm{C}-7), 139.2\left(\mathrm{C}-4^{\mathrm{A}}\right), 140.3$ $\left(\mathrm{C}-4^{\mathrm{D}}\right), 140.4(\mathrm{C}-8 \mathrm{a}), 143.0\left(\mathrm{C}-4^{\mathrm{C}}\right), 148.3\left(\mathrm{C}-2^{\mathrm{D}}\right), 149.0$ $\left(\mathrm{C}-6^{\mathrm{D}}\right), 161.8(\mathrm{COOH}), 166.7(\mathrm{C}-2), 187.9(\mathrm{C}-4) ;{ }^{15} \mathrm{~N}$ NMR $\left(51 \mathrm{MHz}, \mathrm{DMSO}-d_{6}\right) \delta 137.8(\mathrm{~N}-1), 249.7\left(\mathrm{~N}-1^{\mathrm{A}}\right)$, $260.4\left(\mathrm{~N}-1^{\mathrm{C}}\right), 284.9\left(\mathrm{~N}-1^{\mathrm{D}}\right), 356.8\left(\mathrm{~N}-3^{\mathrm{A}}\right), 357.1\left(\mathrm{~N}-3^{\mathrm{C}}\right)$; IR $\left(\mathrm{cm}^{-1}\right)$ : v 3435, 3157, 2927, 1718, 1682, 1600, 1470, 1375, $1313,1189,1035,779,758,696$; HRMS (ESI+): $m / z$ calcd for $\mathrm{C}_{26} \mathrm{H}_{19} \mathrm{~N}_{8} \mathrm{O}_{4}{ }^{+}[\mathrm{M}+\mathrm{H}]^{+}$507.1524, found 507.1527.

\section{Acknowledgments.}

This work was financed by TBU in Zlín (internal grant no. IGA/FT/2019/010, funded from the resources of specific university research). The authors acknowledge also the financial support from the Slovenian Research Agency (Research Core Funding Grant P1-0230, Project J1-8147, and Project J1-9166).

\section{References}

1. D. Urankar, B. Pinter, A. Pevec, F. De Proft, I. Turel, J. Košmrlj, Inorg. Chem. 2010, 49, 4820-4829. DOI:10.1021/ic902354e
2. K. J. Kilpin, E. L. Gavey, C. J. McAdam, C. B. Anderson, S. J. Lind, C. C. Keep, K. C. Gordon, J. D. Crowley, Inorg Chem 2011, 50, 6334-6346. DOI:10.1021/ic200789b

3. J. D. Crowley, D. A. McMorran, Top. Heterocycl. Chem. 2012, 28,31-84. DOI:10.1007/7081_2011_67

4. R. J. Detz, S. A. Heras, R. de Gelder, P. W. N. M. Van Leeuwen, H. Hiemstra, J. N. H. Reek, J. H. Van Maarseveen, Org. Lett. 2006, 8, 3227-3230. DOI:10.1021/ol061015q

5. B. Beyer, C. Ulbricht, D. Escudero, C. Friebe, A. Winter, L. González, U. S. Schubert, Organometallics 2009, 28, 54785498. DOI:10.1021/om9003785

6. A. Kumar, R. K. Chhatra, P. S. Pandey, Org. Lett. 2010, 12, 24-27. DOI:10.1021/ol902351g

7. S. H. Kim, H. S. Choi, J. Kim, S. J. Lee, D. T. Quang, J. S. Kim, Org. Lett. 2010, 12, 560-563. DOI:10.1021/ol902743s

8. J. M. Fernández-Hernández, C. H. Yang, J. I. Beltrán, V. Lemaur, F. Polo, R. Fröhlich, J. Cornil, L. De Cola, J. Am. Chem. Soc. 2011, 133, 10543-10558.

DOI: $10.1021 /$ ja201691b

9. Y. C. Duan, Y. C. Ma, E. Zhang, X. J. Shi, M. M. Wang, X. W. Ye, H. M. Liu, Eur. J. Med. Chem. 2013, 62, 11-19.

DOI:10.1016/j.ejmech.2012.12.046

10. B. Garudachari, A. M. Isloor, M. N. Satyanarayana, H.K. Fun, G. Hegde, Eur. J. Med. Chem. 2014, 74, 324-332. DOI:10.1016/j.ejmech.2014.01.008

11. Y. W. He, C. Z. Dong, J. Y. Zhao, L. L. Ma, Y. H. Li, H. A. Aisa, Eur. J. Med. Chem. 2014, 76, 245-255.

DOI:10.1016/j.ejmech.2014.02.029

12. K. T. Petrova, T. M. Potewar, P. Correira-da-Silva, M. T. Barros, R. C. Calhelha, A. Ćiric, M. Soković, I. C. F. R. Ferreira, Carbohydr. Res. 2015, 417, 66-71.

DOI:10.1016/j.carres.2015.09.003

13. I. Fichtali, W. Laaboudi, E. M. El Hadrami, F. El Aroussi, A. Ben-Tama, M. Benlemlih, S. E. Stiriba, J. Mater. Environ. Sci. 2016, 7, 1633-1641.

14. M. R. Jones, E. Mathieu, C. Dyrager, S. Faissner, Z. Vaillancourt, K. J. Korshavn, M. H. Lim, A. Ramamoorthy, V. W. Yong, S. Tsutsui, P. K. Stys, T. Storr, Chem. Sci. 2017, 8, 56365643. DOI:10.1039/C7SC01269A

15. A. Bolje, J. Košmrlj, Org. Lett. 2013, 15, 5084-5087. DOI:10.1021/ol4024584

16. A. Bolje, S. Hohloch, D. Urankar, A. Pevec, M. Gazvoda, B. Sarkar, J. Košmrlj, Organometallics 2014, 33, 2588-2598. DOI:10.1021/om500287t

17. S. Hohloch, S. Kaiser, F. L. Duecker, A. Bolje, R. Maity, J. Košmrlj, B. Sarkar, Dalton Trans. 2015, 44, 686-693. DOI:10.1039/C4DT02879A

18. M. Gazvoda, M. Virant, A. Pevec, D. Urankar, A. Bolje, M. Kočevar, J. Košmrlj, Chem. Commun. (Cambridge, U. K.) 2016, 52, 1571-1574. DOI:10.1039/C5CC08717A

19. K. Proisl, S. Kafka, J. Košmrlj, Curr. Org. Chem. 2017, 21, 1949-1975. DOI:10.2174/1385272821666170711155631

20. S. Kafka, S. Hauke, A. Salčinović, O. Soidinsalo, D. Urankar, J. Košmrlj, Molecules 2011, 16, 4070-4081.

DOI: $10.3390 /$ molecules 16054070

21. A. C.-M. Daugan, Y. Lamotte, O. Mirguet, Quinolinone de- 
rivatives, PCT Int Appl WO 2012/119978, date of patent September 13, 2012.

22. N. P. Grimster, B. Stump, J. R. Fotsing, T. Weide, T. T. Talley, J. G. Yamauchi, A. Nemecz, C. Kim, K.-Y. Ho, K. B. Sharpless, P. Taylor, V. V. Fokin, J. Am. Chem. Soc. 2012, 134, 6732-6740. DOI:10.1021/ja3001858

23. J. G. Yamauchi, K. Gomez, N. Grimster, M. Dufouil, A. Nemecz, J. R. Fotsing, K.-Y. Ho, T. T. Talley, K. B. Sharpless, V. V. Fokin, P. Taylor, Mol. Pharmacol. 2012, 82, 687-699. DOI:10.1124/mol.112.080291

24. C. D. Rillahan, E. Schwartz, R. McBride, V. V. Fokin, J. C. Paulson, Angew. Chem. Int. Ed. 2012, 51, 11014-11018. DOI:10.1002/anie.201205831

25. Y. Miyamoto, J. Kalisiak, K. Korthals, T. Lauwaet, D. Y. Cheung, R. Lozano, E. R. Cobo, P. Upcroft, J. A. Upcroft, D. E. Berg, F. D. Gillin, V. V. Fokin, K. B. Sharpless, L. Eckmann, Proc. Natl. Acad. Sci. U. S. A. 2013, 110, 17564-17569. DOI:10.1073/pnas.1302664110

26. J. S. Oakdale, R. K. Sit, V. V. Fokin, Chem. - Eur. J. 2014, 20, 11101-11110. DOI:10.1002/chem.201402559

27. V. Fokin, B. K. Sharpless, L. Eckmann, Y. Miyamoto, Expanded therapeutic potential in nitroheteroaryl antimicrobials, PCT Int Appl WO 2014/205414, date of patent December 24, 2014.

28. J. Dong, K. B. Sharpless, Sulfur(VI) fluoride compounds and methods for the preparation thereof, PCT Int Appl WO 2015/188120, date of patent December 10, 2015.

29. W. J. Kim, K. A. Korthals, S. Li, C. Le, J. Kalisiak, K. B. Sharpless, V. V. Fokin, Y. Miyamoto, L. Eckmann, Antimicrob. Agents Chemother. 2017, 61, e02397/1- e02397/12.

DOI:10.1128/AAC.02397-16.

30. D. Milićević, R. Kimmel, M. Gazvoda, D. Urankar, S. Kafka, J. Košmrlj, Molecules 2018, 23, 2310/1-2310/21.

DOI:10.3390/molecules23092310

31. N. Boechat, V. F. Ferreira, S. B. Ferreira, M. de L. G. Ferreira, Da F. de C. Silva, M. M. Bastos, M. dos S. Costa, M. C. S. Lourenço, A. C. Pinto, A. U. Krettli, A. C. Aguiar, B. M. Teixeira, Da N. V. Silva, P. R. C. Martins, F. A. F. M. Bezerra, A. L. S. Camilo, G. P. Da Silva, C. C. P. Costa, J. Med. Chem. 2011,

\section{4, 5988-5999. DOI:10.1021/jm2003624}

32. D. M. Reddy, J. Srinivas, G. Chashoo, A. K. Saxena, H. M. S. Kumar, Eur. J. Med. Chem. 2011, 46, 1983-1991.

DOI:10.1016/j.ejmech.2011.02.016

33. M. Aufort, J. Herscovici, P. Bouhours, N. Moreau, C. Girard, Bioorg. Med. Chem. Lett. 2008, 18, 1195-1198.

DOI:10.1016/j.bmcl.2007.11.111

34. P. R. Bagdi, R. S. Basha, A. T. Khan, RSC Adv. 2015, 5, 6133761344. DOI:10.1039/C5RA09671E

35. B. Orzeszko, M. Fedoryński, A. E. Laudy, B. J. Starościak, A. Orzeszko, Acta Pol. Pharm. 2006, 63, 374-377.

36. T. Sasaki, S. Eguchi, M. Yamaguchi, T. Esaki, J. Org. Chem. 1981, 46, 1800-1804. DOI:10.1021/jo00322a009

37. X. Wang, Z.-C. Dai, Y.-F. Chen, L.-L. Cao, W. Yan, S.-K. Li, J.X. Wang, Z.-G. Zhang, Y.-H. Ye, Eur. J. Med. Chem. 2017, 126, 171-182. DOI:10.1016/j.ejmech.2016.10.006

38. C. Han, S. Dong, W. Zhang, Z. Chen, Synlett 2018, 29, 673677. DOI:10.1055/s-0036-1589157

39. M. N. Rajadhyaksha, R. Nair, P. V. Ramesan, K. Johnson, A. M. Panandikar, Process for preparation of fluorinated triazole compound, PCT Int Appl WO 2012/032540, date of patent March 15, 2012.

40. Y. Naro, N. Ankenbruck, M. Thomas, Y. Tivon, C. M. Connelly, L. Gardner, A. Deiters, J. Med. Chem. 2018, 61, 5900-5909. DOI:10.1021/acs.jmedchem.7b01891

41. J. M. Rieger, M. L. Brown, G. W. Sullivan, J. Linden, T. L. Macdonald, J. Med. Chem. 2001, 44, 531-539. DOI:10.1021/jm0003642

42. G. Abbiati, A. Contini, D. Nava, E. Rossi, Tetrahedron 2009, 65, 4664-4670. DOI:10.1016/j.tet.2009.04.041

43. D.-S. Kim, K. Bolla, S. Lee, J. Ham, Tetrahedron 2011, 67, 1062-1070. DOI:10.1016/j.tet.2010.12.049

44. Oxford Diffraction, CrysAlis PRO, Oxford Diffraction Ltd., Yarnton, England, 2009.

45. G. M. Sheldrick, Acta Crystallogr., Sect. A: Found. Crystallogr. 2008, 64, 112-122. DOI:10.1107/S0108767307043930

46. G. M. Sheldrick, Acta Crystallogr., Sect. C: Struct. Chem. 2015, 71,3-8. DOI:10.1107/S2053229614024218

\section{Povzetek}

V prispevku je opisana kisla etanoliza (deacetiliranje) (1-(2,4-diokso-1,2,3,4-tetrahidrokinolin-3-il)-1H-1,2,3-triazol-4-il)metil acetatov, substituiranih na dušikovem atomu kinolindionske skupine s propargilno skupino ali pa $\mathrm{z}$ (1-substituirano $1 H$-1,2,3-triazol-4-il)metilno skupino. Izhodni acetate so pripravili iz ustreznih 3-(4-hidroksimetil-1H-1,2,3-triazol-1-il)kinolin-2,4(1H,3H)-dionov, ki niso substituirani na kinolonskem dušiku, po še opisanih postopkih. Tako dobljene primarne alkohole, kot tudi tiste, ki niso substituirani na kinolonskem dušiku, so oksidirali bodisi $\mathrm{v}$ aldehide s piridinijevim klorokromatom (PCC), ali pa $\mathrm{z}$ manganovim dioksidom $\mathrm{v}$ karboksilne kisline, ob uporabi Jones-ovega reagent $\mathrm{v}$ acetonu kot topilu. Strukture vseh pripravljenih spojin so potrdili $\mathrm{z}{ }^{1} \mathrm{H},{ }^{13} \mathrm{C}$ and ${ }^{15} \mathrm{~N}$ NMR spectroskopijo. Ustrezne rešitve struktur analiziranih spojin so bile narejene na podlagi standardnih $1 \mathrm{D}$ in izbranih gradientnih 2D NMR poskusov $\left({ }^{1} \mathrm{H}-{ }^{1} \mathrm{H}\right.$ gs-COSY, ${ }^{1} \mathrm{H}-{ }^{13} \mathrm{C}$ gs-HSQC, ${ }^{1} \mathrm{H}-{ }^{13} \mathrm{C}$ gs-HMBC), skupaj z ${ }^{1} \mathrm{H}-{ }^{15} \mathrm{~N}$ gs- $\mathrm{HMBC}$, kot praktičnim orodjem za določitev ${ }^{15} \mathrm{~N}$ NMR kemijskih premikov v spojinah, ki niso obogatene $\mathrm{z}^{15} \mathrm{~N}$ izotopom.

Except when otherwise noted, articles in this journal are published under the terms and conditions of the Creative Commons Attribution 4.0 International License 